\title{
Stabilization of desmoglein-2 binding rescues arrhythmia in arrhythmogenic cardiomyopathy
}

\author{
Camilla Schinner, ${ }^{1,2}$ Bernd Markus Erber, ${ }^{1}$ Sunil Yeruva, ${ }^{1}$ Angela Schlipp, ${ }^{1}$ Vera Rötzer, ${ }^{1}$ Ellen Kempf, ${ }^{1}$ \\ Sebastian Kant, ${ }^{3}$ Rudolf E. Leube, ${ }^{3}$ Thomas D. Mueller, ${ }^{4}$ and Jens Waschke ${ }^{1}$ \\ 'Faculty of Medicine, Ludwig-Maximilians-Universität (LMU) Munich, Munich, Germany. 'Department of Biomedicine, \\ University of Basel, Basel, Switzerland. ${ }^{3}$ nnstitute of Molecular and Cellular Anatomy, RWTH Aachen University, Aachen, \\ Germany. ${ }^{4}$ Department of Molecular Plant Physiology and Biophysics, Julius-von-Sachs Institute for Biosciences, Julius- \\ Maximilians-Universität, Würzburg, Germany.
}

\begin{abstract}
Arrhythmogenic cardiomyopathy $(\mathrm{AC})$ is a genetic disease causing arrhythmia and sudden cardiac death with only symptomatic therapy available at present. Mutations of desmosomal proteins, including desmoglein-2 (Dsg2) and plakoglobin ( $\mathrm{Pg})$, are the major cause of $A C$ and have been shown to lead to impaired gap junction function. Recent data indicated the involvement of antiDsg2 autoantibodies in AC pathogenesis. We applied a peptide to stabilize Dsg2 binding similar to a translational approach to pemphigus, which is caused by anti-desmoglein autoantibodies. We provide evidence that stabilization of Dsg2 binding by a linking peptide (Dsg2-LP) is efficient to rescue arrhythmia in an $A C$ mouse model immediately upon perfusion. Dsg2-LP, designed to cross-link Dsg2 molecules in proximity to the known binding pocket, stabilized Dsg2-mediated interactions on the surface of living cardiomyocytes as revealed by atomic force microscopy and induced Dsg2 oligomerization. Moreover, Dsg2-LP rescued disrupted cohesion induced by siRNAmediated Pg or Dsg2 depletion or L-tryptophan, which was applied to impair overall cadherin binding. Dsg2-LP rescued connexin-43 mislocalization and conduction irregularities in response to impaired cardiomyocyte cohesion. These results demonstrate that stabilization of Dsg2 binding by Dsg2-LP can serve as a novel approach to treat arrhythmia in patients with AC.
\end{abstract}

Authorship note: CS, BME, and SY contributed equally to this work.

Conflict of interest: The authors have declared that no conflict of interest exists.

Copyright: () 2020, American Society for Clinical Investigation.

Submitted: May 10, 2019

Accepted: April 8, 2020

Published: May 7, 2020.

Reference information: /CI Insight. 2020;5(9):e130141.

https://doi.org/10.1172/jici.

insight.130141.

\section{Introduction}

Arrhythmogenic cardiomyopathy (AC) is a hereditary disease with a high risk for sudden cardiac death at a young age. Several desmosomal protein-encoding genes, such as for plakophilin-2 (Pkp2), desmoplakin (Dp), plakoglobin (Pg), desmocollin-2 (Dsc2), and desmoglein-2 (Dsg2), as well as a few nondesmosomal genes can be affected by mutations. However, it is not entirely clear how alterations in the proteins encoded by these genes contribute to arrhythmia, cardiac fibrosis, and subsequent heart failure in patients with AC. Thus, at present only symptomatic therapy is available (1). However, a recent study demonstrated antiDsg2 autoantibodies to be present in patients with AC independent of the underlying mutation. These antibodies were capable of inducing an impairment of gap junction function (2).

Desmosomes, adherens junctions (AJ), and gap junctions (GJ) form the intercalated disk (ICD), a structural and functional unit providing electrical conduction and mechanical stability in the heart. Connection between 2 adjacent cells is provided by members of the cadherin family, which span the intercellular cleft and are linked to several plaque proteins and the cytoskeleton. Dsg2 and Dsc2 are the desmosomal cadherins expressed in cardiomyocytes, while $\mathrm{N}$-cadherin $(\mathrm{N}-\mathrm{Cad})$ is found in cardiac AJ (3). As is known for cadherins in general, the extracellular binding seems to depend on the so-called Trp swap, where an L-tryptophan ( $\operatorname{Trp})$ residue at position 2 of 1 cadherin extracellular domain fits into a hydrophobic pocket of an adjacent cadherin and vice versa (4). On the cytoplasmic side of cardiomyocytes, desmosomal adhesion molecules are anchored to the desmin cytoskeleton via Pkp2, Dp, and Pg (3).

GJs of cardiomyocytes mainly consist of connexin $43(\mathrm{Cx} 43)$ and facilitate electrical current flow for coordinated contraction as well as intercellular passage of ions and small molecules. They are subjected to fast and variable modifications, such as phosphorylation, to regulate conduction velocity and channel 
permeability (5). For instance, phosphorylation of Cx43 at serine 368 (Ser368) by PKC protects cardiac tissue from ischemic damage via attenuation of GJ communication $(6,7)$. Interestingly, PKC signaling is also important for modulation of desmosomal function in cardiomyocytes (8). It is accepted that desmosomal and AJ components are required for maintenance of electrical coupling of cardiomyocytes because multiple studies demonstrated the impairment of GJ function and distribution by disrupted mechanical strength of the ICD (8-12). Following from this, improvement of cardiomyocyte cohesion may be effective to rescue impaired electrical conduction. Furthermore, a peptide-based stabilization of intercellular cohesion was shown to be protective in the autoimmune disease pemphigus (13), where autoantibodies against Dsg3 impair keratinocyte cohesion with induction of skin blistering (14). Thus, an analog approach could serve as a novel therapeutic target for treating arrhythmias in AC.

In this study, we demonstrate for the first time to our knowledge that stabilization of Dsg2-mediated binding by a specific linking peptide (Dsg2-LP) is capable of rescuing both cardiomyocyte cohesion and GJ-mediated signal transduction caused by disruption of intercellular adhesion. Stabilization of Dsg2 binding improved GJ communication in a PKC-dependent manner, which was paralleled by phosphorylation of Cx43 at Ser368. Because arrhythmia in perfused hearts derived from an AC mouse model was successfully reduced by Dsg2-LP treatment, these results indicate that stabilization of Dsg2 binding can be an effective approach to treat AC.

\section{Results}

Dsg2-LP reduces arrhythmia in an AC mouse model. Using data of crystal structure analyses, we designed a new linking peptide to stabilize 2 interacting Dsg2 molecules, similar to reports for Dsg3 and VE-cadherin $(13,15)$ (Figure 1, A-C). Our model is based on the crystal structures of N-Cad (16), which are arranged in different assemblies mimicking cis and trans interaction and are consistent with recent structures of Dsg2 (17). Dsg2-LP was designed as a dimer of 2 cyclic peptides to mimic the predicted cis-interacting peptide sequence of 2 extracellular 1 domains and thus to cross-link Dsg2 molecules in proximity to the binding pocket harboring the Trp swap.

To investigate the effects of Dsg2-LP on hearts exhibiting an AC phenotype, we performed ex vivo studies on perfused hearts from an AC mouse model with cardiac deficiency of the desmosomal plaque protein Pg (Pg-KO), which we established recently (18). Similar to hearts of patients with AC, hearts of Pg-KO mice show arrhythmias with ventricular dilatation, fibrosis, and hypertrophy. To test whether Dsg2-LP is effective to reduce arrhythmia in this AC model, heart rate was evaluated by ECG at baseline with subsequent Dsg2-LP perfusion for 20 minutes. As a control for Dsg2-LP, a similar peptide but specific for linking VE-cadherin extracellular domains (VE-Cad-LP) was used, which was shown not to interfere with desmosomal binding (15). In this setup, the degree of arrhythmia was objectified by calculating the duration of all R-R intervals and the standard deviation of these values (SDNN) under baseline conditions and 10 minutes after treatment. Perfusion with Dsg2-LP significantly reduced arrhythmia as revealed by a significant drop of the SDNN in Pg-KO hearts (Figure 1, D and E). In contrast, perfusion of Pg-KO hearts with VE-Cad-LP did not show a protective effect on SDNN (Figure $1, \mathrm{~F}$ and $\mathrm{G}$ ). In comparison, the SDNN of WT littermates' hearts was not affected by perfusion with Dsg2-LP (Figure 1, H and I). In addition, we measured pulse pressure and heart rate, which were not altered significantly after Dsg2-LP or VE-Cad-LP treatment in Pg-KO or WT hearts (Supplemental Figure 1; supplemental material available online with this article; https://doi.org/10.1172/jci. insight.130141DS1). These results demonstrate that Dsg2-LP improves arrhythmia in an AC mouse model quickly after peptide application.

Dsg2-LP reorganizes CX43 and Dsg2 and rescues cell cohesion in response to disruption of intercellular junctions. To investigate the underlying mechanisms of how Dsg2-LP can rescue arrhythmia, distribution of Dsg2 and the gap junction protein $\mathrm{Cx} 43$ was simultaneously determined by high-resolution stimulated emission depletion (STED) microscopy in cardiac slice culture of Pg-KO and WT mice after treatment with Dsg2LP for 10 minutes similar to the ex vivo studies shown in Figure 1, D-I. In WT hearts, no obvious changes of Dsg2 or Cx43 signal intensity or colocalization of both signals was induced by Dsg2-LP at the ICD (Figure 2, A and B). In Pg-deficient hearts, Dsg2-LP treatment led to an elevation of Dsg2 at the ICD with an increase of colocalizing pixels to levels comparable to WT. Similar results were obtained after incubation for 1 hour (Supplemental Figure 2). This indicates reorganization of Dsg2 and Cx43 at the ICD by Dsg2-LP in parallel to the reduction of arrhythmia in Pg-deficient hearts. 
A

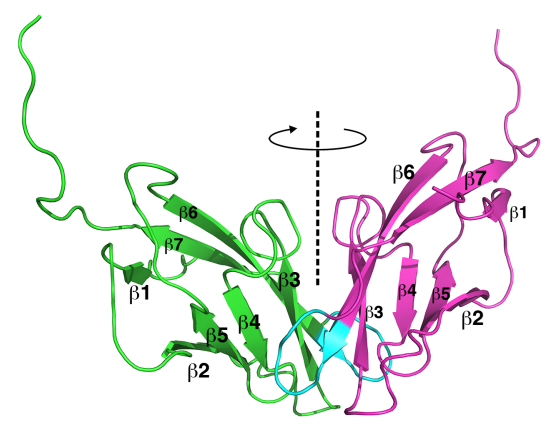

B637-loop
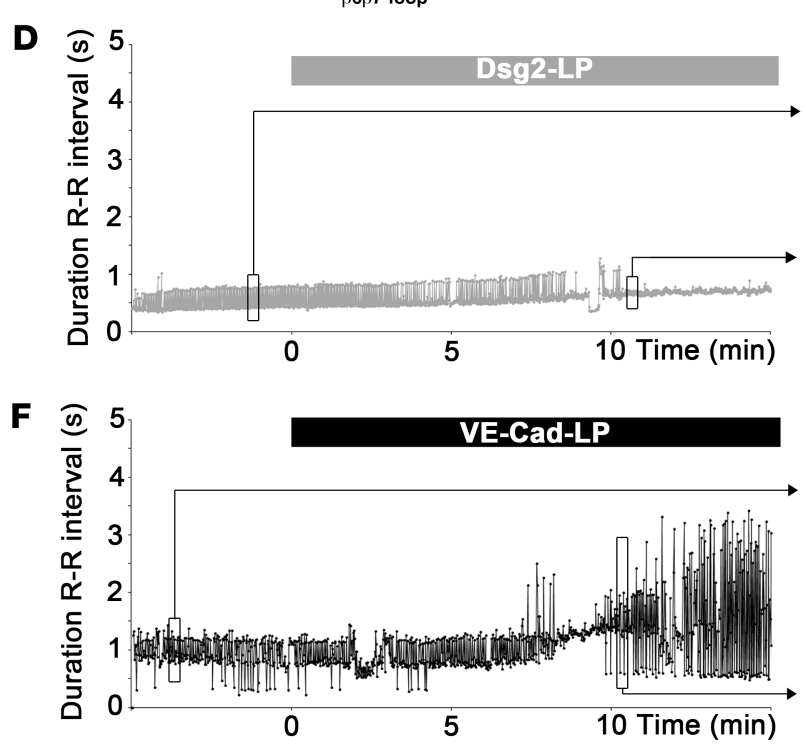

H

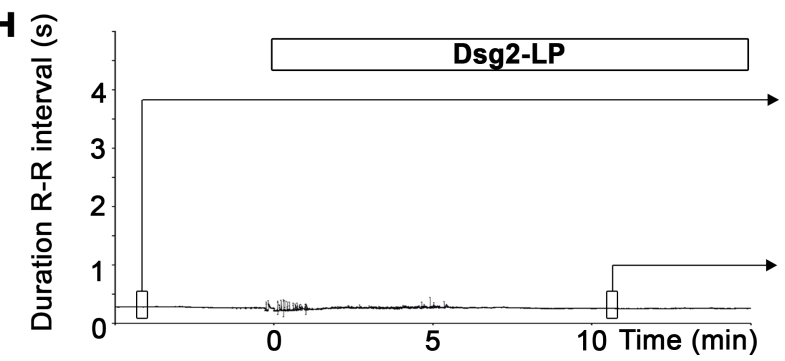

B

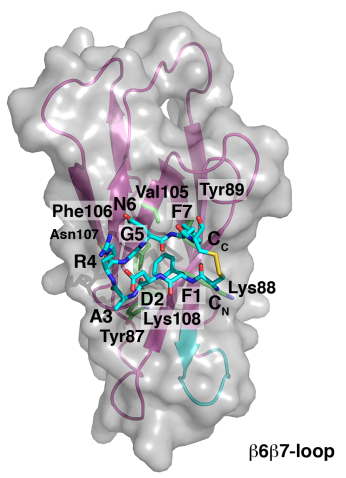

C
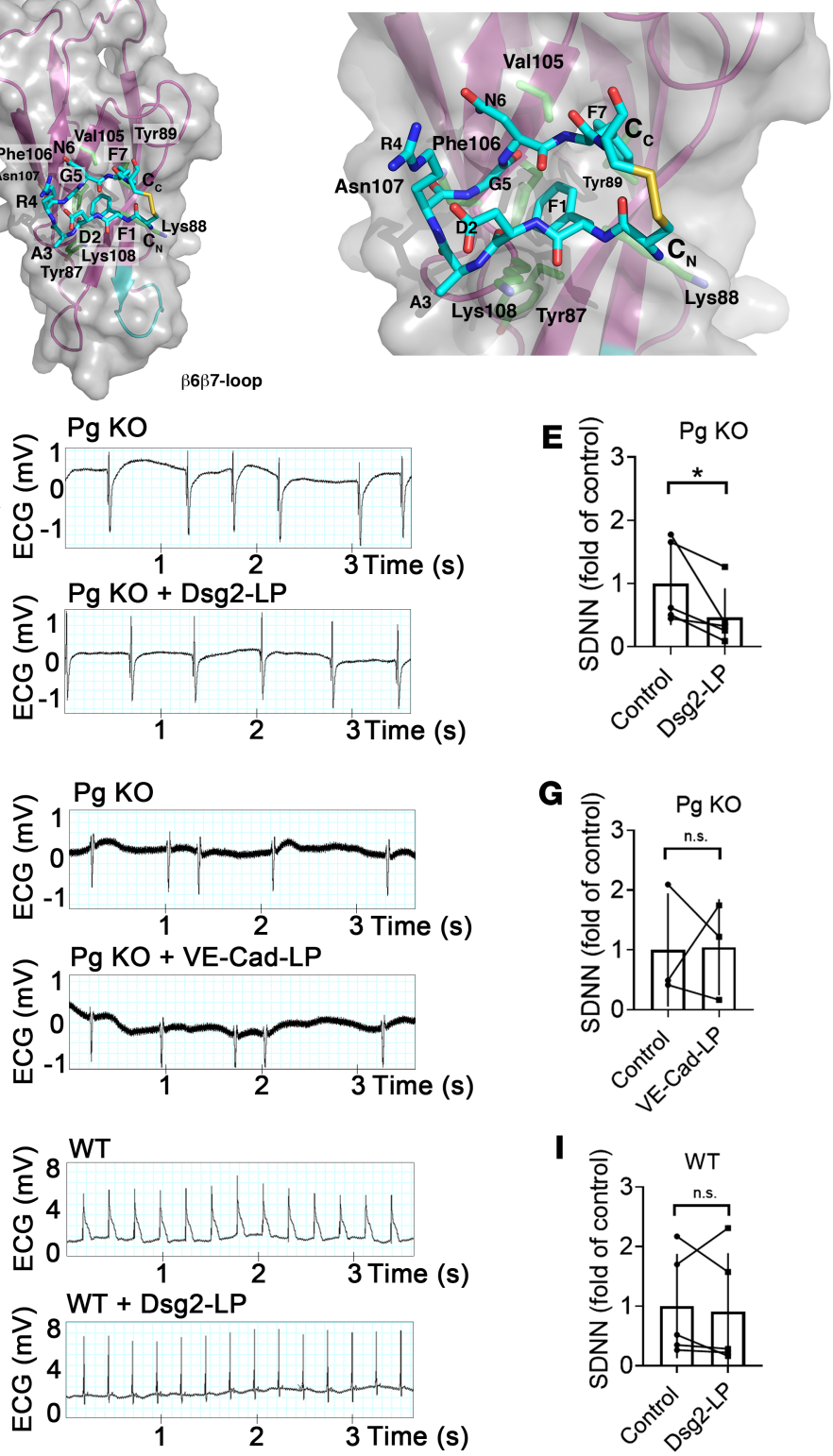
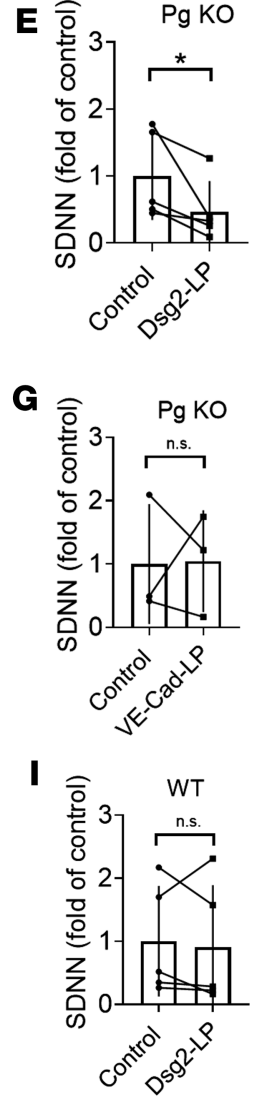

Figure 1. Dsg2-LP reduces arrhythmias in perfused AC-like mouse hearts. (A) Modeling of the potential cis interaction between 2 Dsg2 extracellular 1 domains (EC1s). Dashed line indicates 2-fold symmetry axis of the 2 EC1s. (B) Structure of the monomeric peptide (Dsg2-IP) fitted to the EC1 of Dsg2. Residues potentially in contact with the peptide are shown as sticks and numbered in 3-letter code accordingly. (C) Magnification of B. Dsg2-IP shown; C atoms colored in cyan. Interface residues located on the central $\beta$-sheet are shown as sticks with $\mathbf{C}$ atoms colored in green. ( $\mathbf{D}$ and $\mathbf{F}$ ) Representative time course of duration of R-R intervals of perfused hearts from Pg-KO mice with AC-like phenotype under baseline conditions and after addition of Dsg2-LP. Every dot indicates the duration of 1 beat-to-beat interval plotted against perfusion time. VE-Cad-LP served as peptide control. Right panel shows representative ECG traces from indicated time points. (E and G) Analysis of SDNN derived from Pg-KO hearts treated as described in D and F. (H and I) Representative time course of duration of R-R intervals with analysis of the SDNN of perfused hearts from WT mice under baseline conditions and after addition of Dsg2LP ( $n=5$ mice for Dsg-LP and Pg-KO and WT; $n=3$ mice for VE-Cad-LP) n.s., $P \geq 0.05,{ }^{*} P<0.05$. Two-tailed paired Student's $t$ test with 95\% confidence level was performed. Black lines indicate paired values.

The functional impact of Dsg2-LP on cardiomyocyte cohesion under these conditions was determined by fragmentation of a floating HL-1 cardiomyocyte monolayer after application of defined mechanical shear stress (18). Under basal conditions, depletion of Pg by siRNA (Pg-siRNA), which was accompanied by reduced Dsg2 levels, significantly increased the number of fragments compared with cells transfected with nontargeting siRNA (n.t.-siRNA) (Figure 3, A and B). Direct reduction of Dsg2 (Dsg2-siRNA) induced similar effects, indicating reduced cell-cell adhesion by depletion of both proteins. Under these conditions, Dsg2-LP treatment reduced the number of fragments to basal 
A
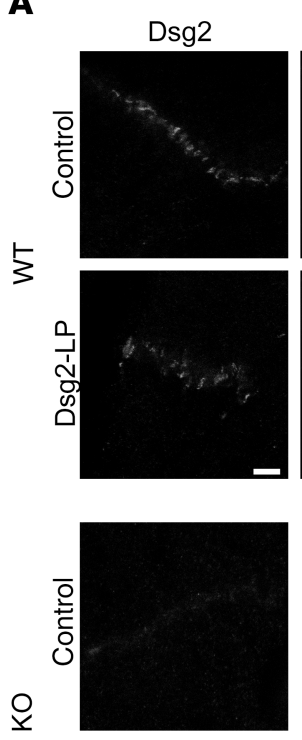

ס

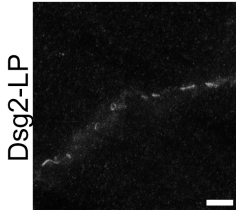

$\mathrm{Cx} 43$
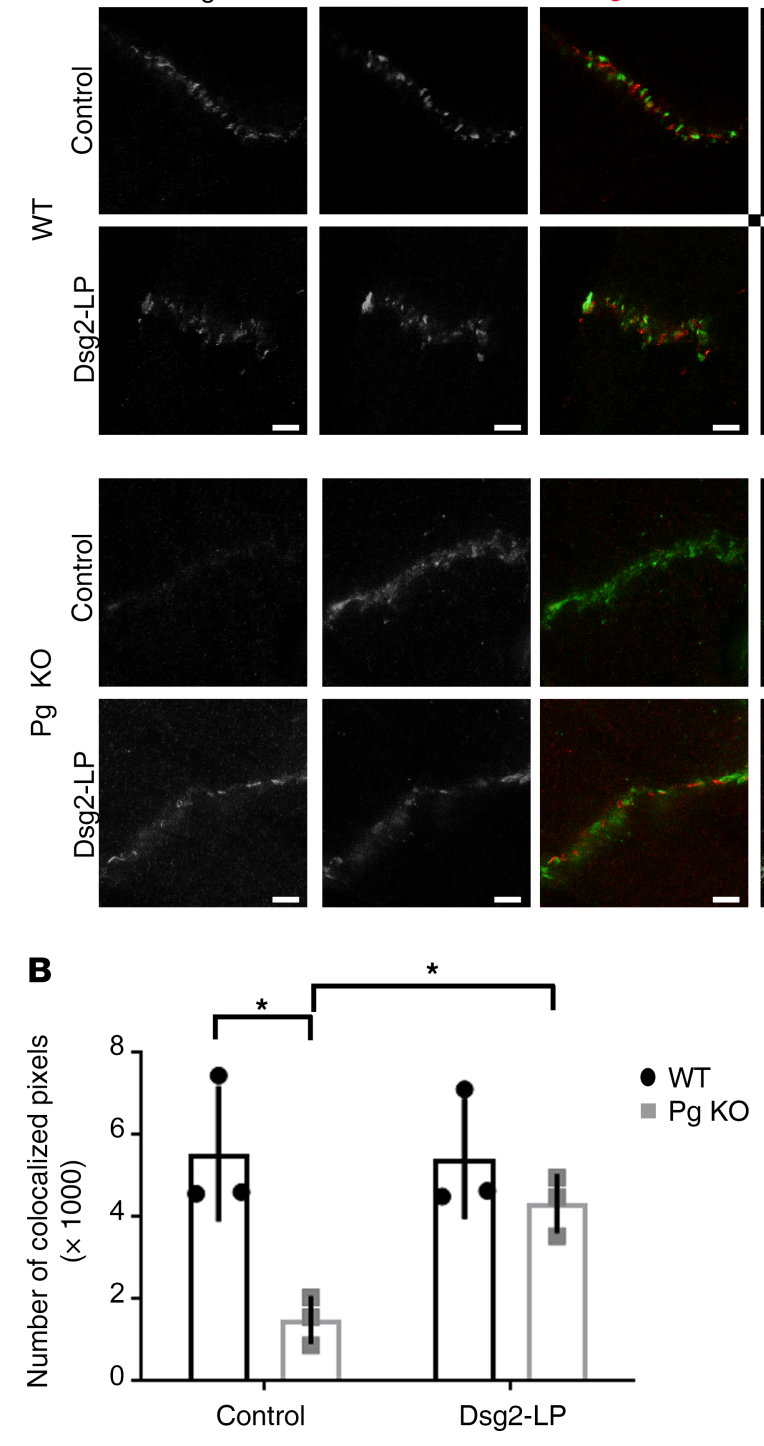

Colocalized

pixels
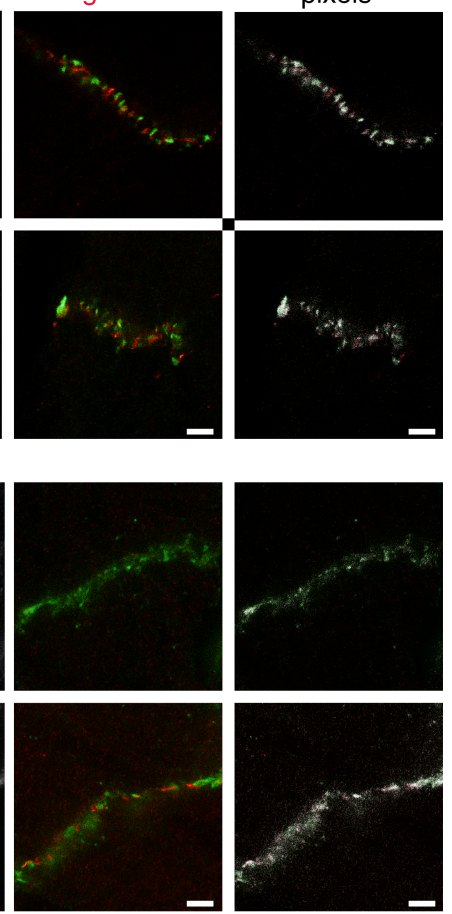

Figure 2. Cx43 membrane localization is rescued by stabilization of Dsg2 binding. (A) STED images of ICDs of WT and Pg-KO mice treated with Dsg2-LP for 10 minutes and stained for Dsg2 (red) and Cx43 (green) (4 ICDs per mouse were analyzed for quantification; $n=3$ mice per condition). Scale bar: $2 \mu \mathrm{m}$. Colocalization of both proteins was analyzed, and colocalized pixels are visualized in white. (B) Quantification of colocalized pixels. ${ }^{*} P<0.05$. Repeated-measures 2-way ANOVA with Holm-Šidák post hoc test was performed.

levels, demonstrating that Dsg2-LP is capable of rescuing cell-cell adhesion in response to desmosomal impairment by reduced levels of Dsg2 or Pg, respectively.

The Trp swap as a common cadherin trans-interacting binding mechanism can be disturbed by an excess of Trp molecules (4). Disruption of cadherin binding by this approach was shown to impair Dsg2 localization and reduce cardiomyocyte cohesion (19). Here, additional Dsg2-LP incubation rescued the reduction of cardiomyocyte cohesion in response to Trp treatment, whereas Dsg2-LP alone did not significantly alter cohesion (Figure 3C). In contrast, specific disruption of Dsg2-mediated binding using an inhibitory peptide (Dsg2-IP) consisting of only the monomeric peptide loop as compared with the 2 linked loops in Dsg2-LP was applied and led to impaired cardiomyocyte cohesion.

To support these findings from cells, beads were coated with Dsg2-Fc and transferred to a hanging drop to form an adhesive layer. After incubation with the indicated mediators, mechanical stress was applied, and results were quantified by calculating the fragmentation index as the ratio of the area of beads per total covered area, with lower values indicating reduced Dsg2-mediated bead-bead adhesion. To confirm the 
A
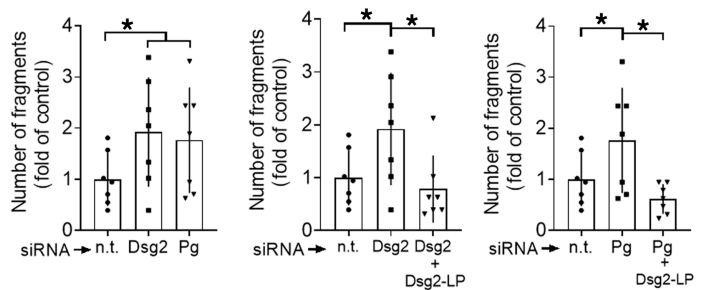

B

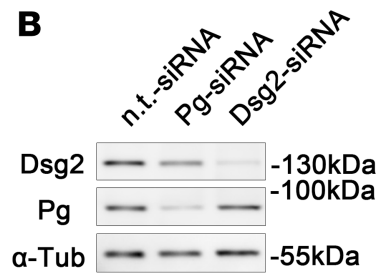

D
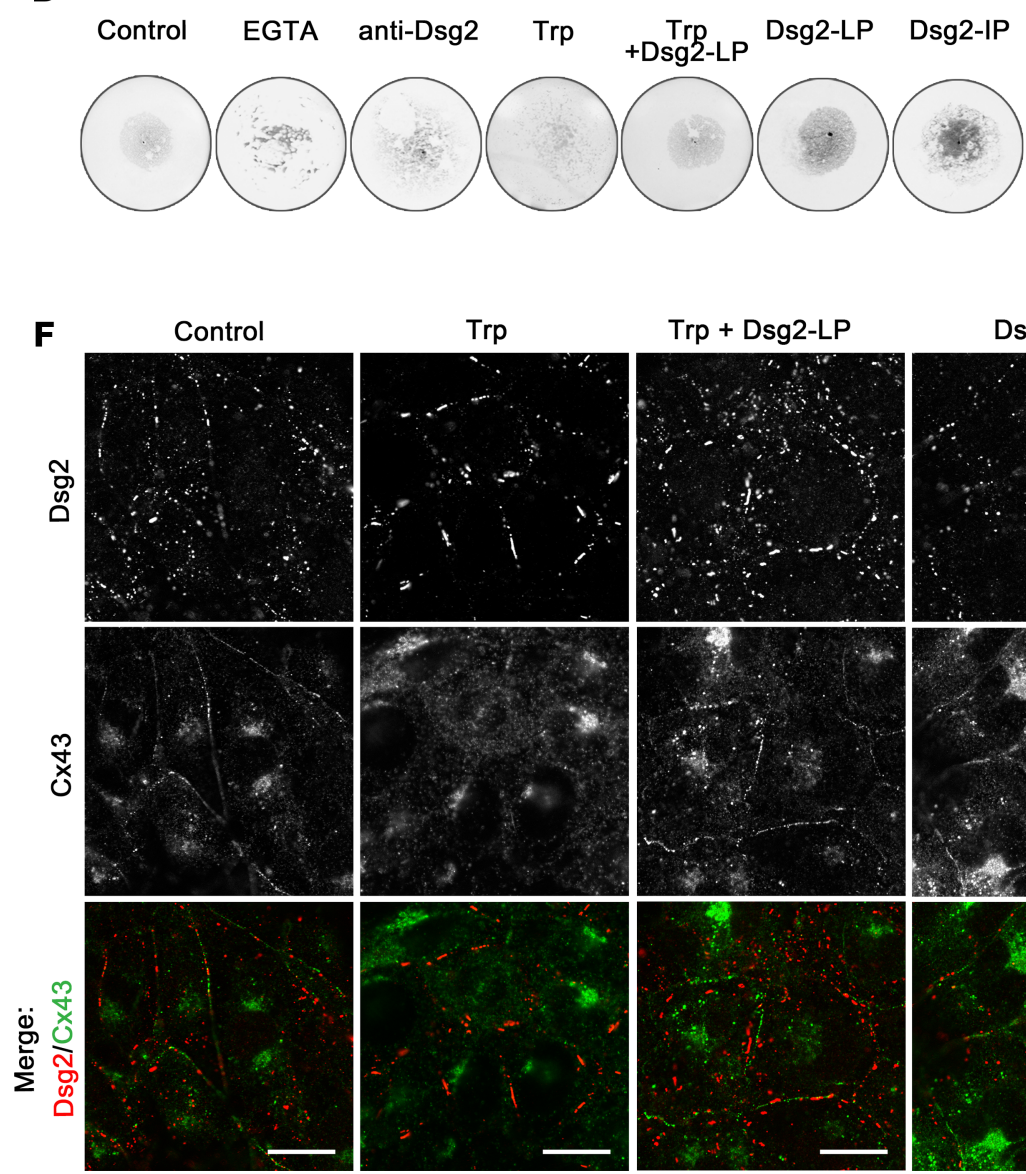

Trp + Dsg2-LP
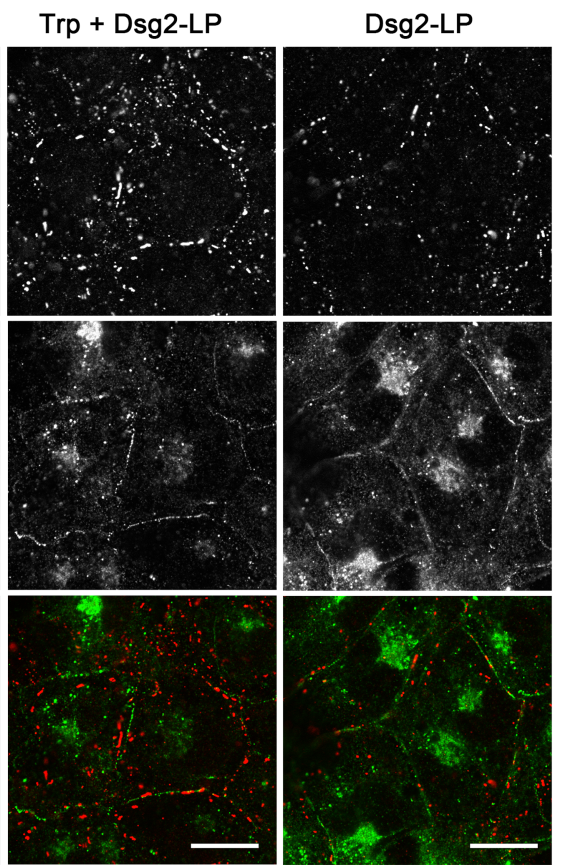

C

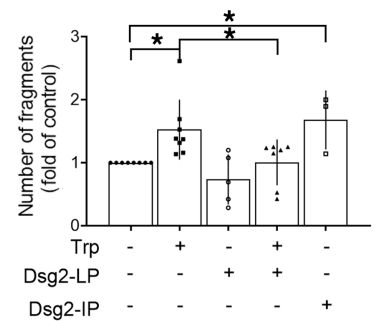

E

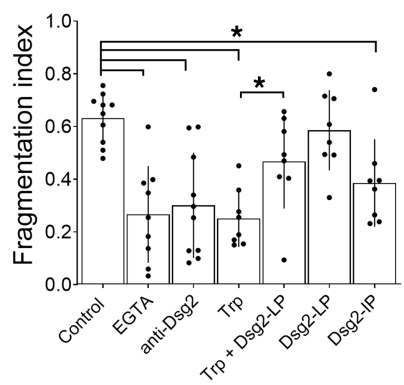

G

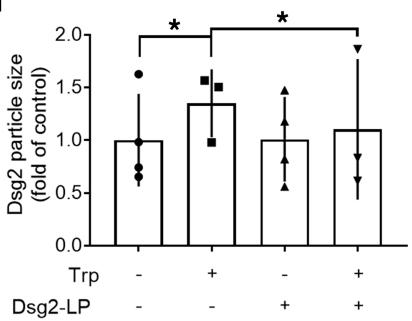

H

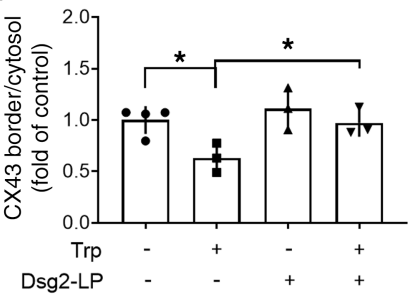

Figure 3. Disruption of intercellular junctions is rescued by stabilization of Dsg2 binding. (A) Dissociation assays of HL-1 monolayer treated with Dsg2- or Pg-siRNA and additional Dsg2-LP treatment $(n=8)$. One-way ANOVA with Bonferroni's post hoc test. (B) Western blot analysis for Dsg2 and Pg performed in parallel to dissociation assays to show successful protein reduction after Dsg2- or Pg-siRNA treatment. $\alpha$-Tubulin ( $\alpha$-Tub) served as loading control ( $n$ = 8). (C) Dissociation assay of HL-1 cell monolayer after disruption of cell cohesion by Trp or Dsg2-IP with Dsg2-LP treatment (control $n=8$; Trp $n=8$; Trp + Dsg-LP $n=7$; Dsg2-LP $n=5$; Dsg2-IP $n=3$ ). One-way ANOVA with Bonferroni's post hoc test. (D) Representative images of bead fragmentation after application of mechanical stress. Adhesion assay of Dsg2-Fc-coated beads with same conditions as C. (E) $\mathrm{Ca}^{2+}$ chelator EGTA and anti-Dsg2 antibody served as negative controls (control $n=10$; EGTA $n=9$; anti-Dsg2 $n=10 ; \operatorname{Trp} n=8$; Trp + Dsg-LP $n=8$; Dsg2-LP $n=8 ;$ Dsg2-IP $n=8$ ). One-way ANOVA with Bonferroni's post hoc test. (F) Representative immunostaining of HL-1 cells stained for Dsg2 (red) and Cx43 (green) treated with Trp, Dsg2-LP, or a combination of both for 24 hours $(n=8)$; scale bar: $10 \mu \mathrm{m}$. (G) Quantitative analysis of single-Dsg2 particle size (>30 cells from 3-4 independent experiments). (H) Quantitative analysis of $\mathrm{Cx} 43$ staining as ratio of intensity of staining at cell-cell border versus cytosol (>30 cells from 3 independent experiments). ${ }^{*} P$ $<0.05$. One-way ANOVA with Tukey's post hoc test.

functionality of this assay, the $\mathrm{Ca}^{2+}$ chelator EGTA was applied to impair cadherin binding, or an inhibitory antibody of Dsg2 interaction (20) was added. Both significantly reduced the fragmentation index, indicating impaired Dsg2-mediated cohesion (Figure 3, D and E). Similarly, Trp excess was effective to disrupt Dsg2 binding, whereas Dsg2-LP incubation was capable of increasing cohesion of beads treated with Trp. Dsg2-LP alone had no effect but blocking Dsg2 interaction by Dsg2-IP significantly reduced Dsg2 binding.

When we evaluated the distribution of Dsg2 and Cx43 in HL-1 cardiomyocytes, Dsg2 immunostaining was detectable at cell-cell contacts in a punctate fashion and partially colocalized with $\mathrm{Cx} 43$, which 
was distributed continuously (Figure 3F). Disruption of cadherin binding by Trp induced accumulation of irregular and enlarged Dsg2 streaks along the membrane, whereas Cx43 staining was significantly diminished at the cell contact area. Additional incubation with Dsg2-LP was effective to prevent Trp-induced disruption of Dsg2 signals and Cx43 localization at the membrane (Figure 3, G and H). However, Dsg2-LP and Trp did not affect protein levels or localization of other desmosomal and AJ proteins, such as Pg, Dp, or N-Cad, as revealed by Western blot analysis and immunostaining in HL-1 cells (Supplemental Figure 3). Altogether, these data demonstrate Dsg2-LP to be effective to restore desmosomal and GJ components at cell contact area and rescue cell cohesion.

Dsg2-LP enhances GJ function via stabilization of Dsg2 binding. Microelectrode arrays (MEAs) were performed to investigate the functional impact of Dsg2-LP on the propagation of excitation and GJ function. Therefore, differences of the extracellular field potential were recorded in cardiac slices of WT and Pg-KO hearts. In WT hearts, spontaneous excitation spread homogeneously from one to the next electrode (Figure 4A). In contrast, Pg-deficient cardiac slices displayed disturbed spreading and delayed conduction of excitation to neighboring areas. Compared with WT slices, the velocity of conduction in Pg-KO was reduced, even though it did not reach statistical significance (Figure 4B). However, Dsg2-LP was efficient to rescue the pattern of excitation conduction and to significantly increase conduction velocity in Pg-KO slices.

Experiments were repeated in HL-1 cultures, where under baseline conditions conduction spread uniformly comparable to WT heart tissue (Figure 4C). As shown before (8), Trp treatment impaired propagation of excitation and caused distinct irregularities with a decrease of conduction velocity and increased amount of arrhythmic beats displayed as elevated SDNN (Figure 4, C-E). Carbenoxolone, a specific GJ inhibitor, induced similar effects, indicating that Trp treatment induced an impairment of GJ function (Supplemental Figure 5). Dsg2-LP suppressed the effect of Trp treatment on conduction velocity as well as on the SDNN. In contrast, neither Dsg2-LP alone nor the inhibitory peptide Dsg2-IP (Supplemental Figure 5) affected conduction of excitation albeit Dsg2-IP but not Dsg2-LP reduced cardiomyocyte cohesion, at least when cells were subjected to mechanical stress. We assume that in the absence of physical load, Dsg2-IP is not sufficient to disturb GJ function because integrity of desmosomal contacts remains intact. Depletion of Dsg2 or Pg in HL-1 cells via siRNA significantly disturbed propagation of excitation with reduced conduction velocity and increased arrhythmic excitation rate (Figure 4, F-H). Importantly, stabilization with Dsg2LP improved arrhythmias under these conditions and increased conduction velocity when Pg was reduced.

It is reported that phosphorylation of Cx43 at Ser368 by PKC regulates conduction velocity and selective permeability of GJ, which has been proposed to protect the myocardium from arrhythmias, for example under ischemic conditions $(6,7)$. It can be envisaged that this mechanism might also be relevant for reducing arrhythmia in response to Dsg2 stabilization. Indeed, in Western blots performed in both HL-1 and ventricular cardiac slices from WT mice, phosphorylation of Cx43 at Ser368 was significantly increased by incubation with Dsg2-LP (Figure 4, I and J). In contrast, in Pg-KO mice, total Cx43 levels were significantly reduced, and phosphorylation of Cx43 was not enhanced by Dsg2-LP (Supplemental Figure 4). This indicates that in the absence of Pg, Dsg2-LP induced Cx43 relocalization, as shown above, without affecting Cx43 levels and phosphorylation. The PKC inhibitor bisindolylmaleimide X (Bim-X) reduced both baseline and enhanced phosphorylation of $\mathrm{Cx} 43$ when used in combination with Dsg2-LP in HL-1 cells and WT heart slices (Figure 4, I and J).

On the functional level, Bim-X coincubation abrogated the effect of Dsg2-LP on velocity of excitation propagation in HL-1 cardiomyocytes with reduced Pg levels (Figure 4, K and L). Similarly, arrhythmia was not significantly rescued by Dsg2-LP under PKC inhibition in HL-1 cells (Figure 4M).

These data indicate that the protective effects of Dsg2-LP on GJ function are at least in part mediated by PKC, which may involve phosphorylation of Cx43 at Ser368.

Dsg2-LP enhances binding force of Dsg2-mediated interactions in vitro. To confirm stabilization of Dsg2 binding by Dsg2-LP, atomic force microscopy (AFM) was applied. A flexible cantilever is functionalized with the extracellular domains of Dsg2 or N-cadherin and repeatedly extended to the surface of a functionalized mica sheet (Figure 5A). During retraction of the cantilever, binding events between the tip-linked proteins and molecules on the mica surface are detectable by scanning the deflection of the tip using a laser beam. The force required to rupture these interactions can be determined, representing the protein interaction strength (21) (Figure 5A). Dsg2-LP was sufficient to increase the binding force between Dsg2 molecules without alteration of the binding frequency (Figure $5 \mathrm{~B}$ ). In contrast, binding force and frequency of $\mathrm{N}$-cadherin interactions were not affected (Figure 5C). 
A
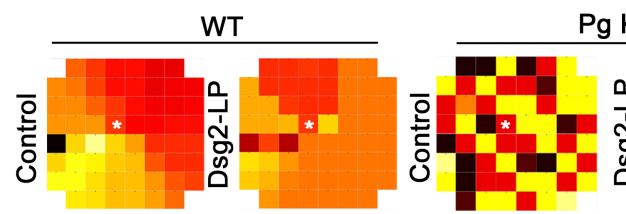

Pg KO

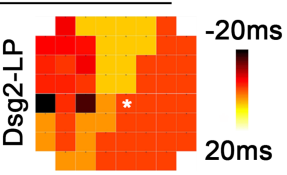

C
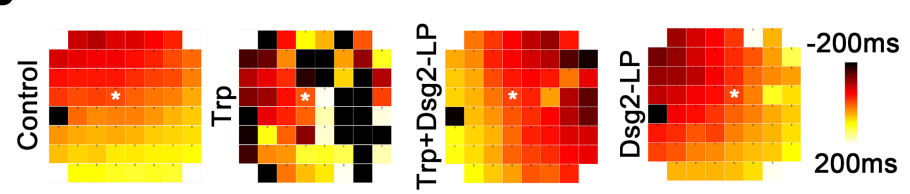

$\mathbf{F}$
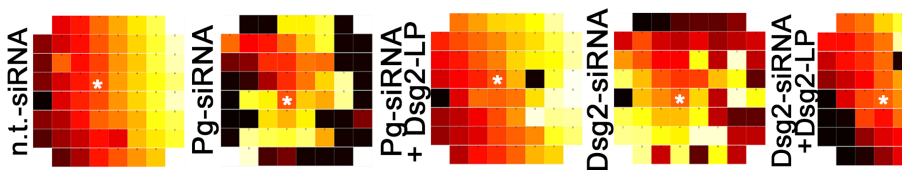

H
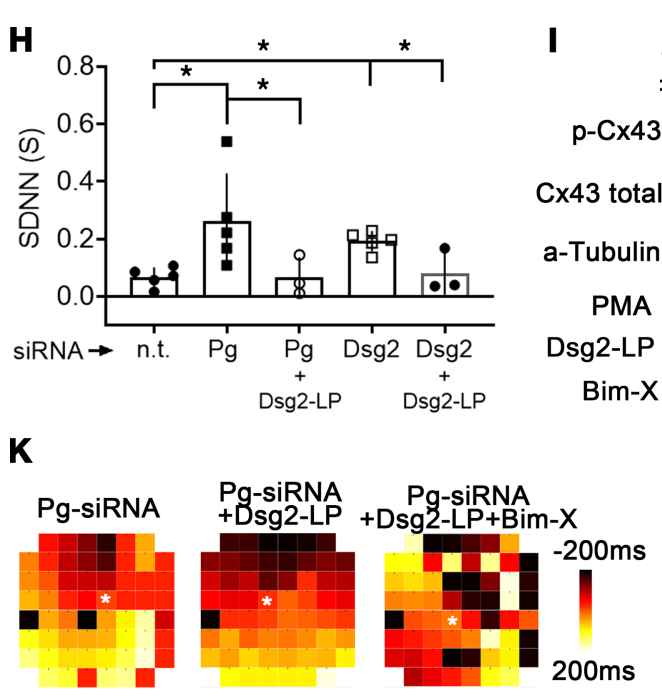
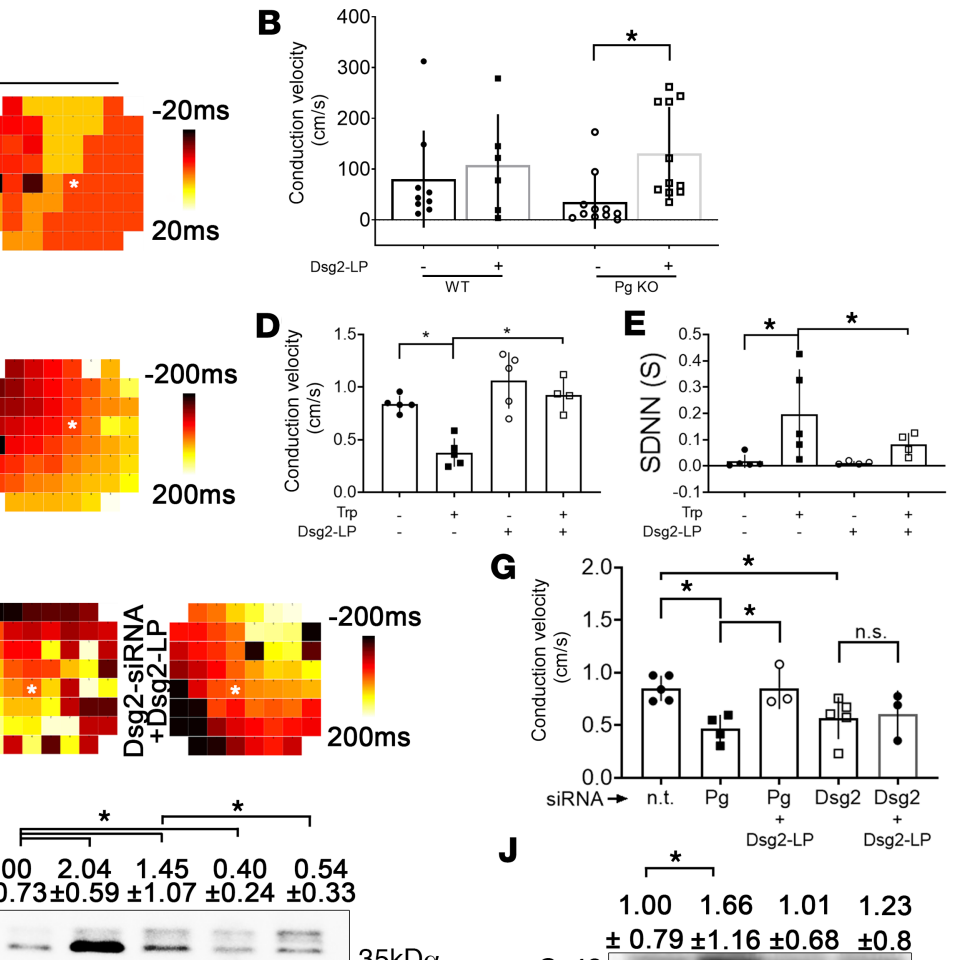

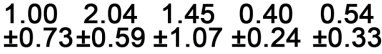

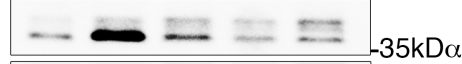

$200 \mathrm{~ms}$
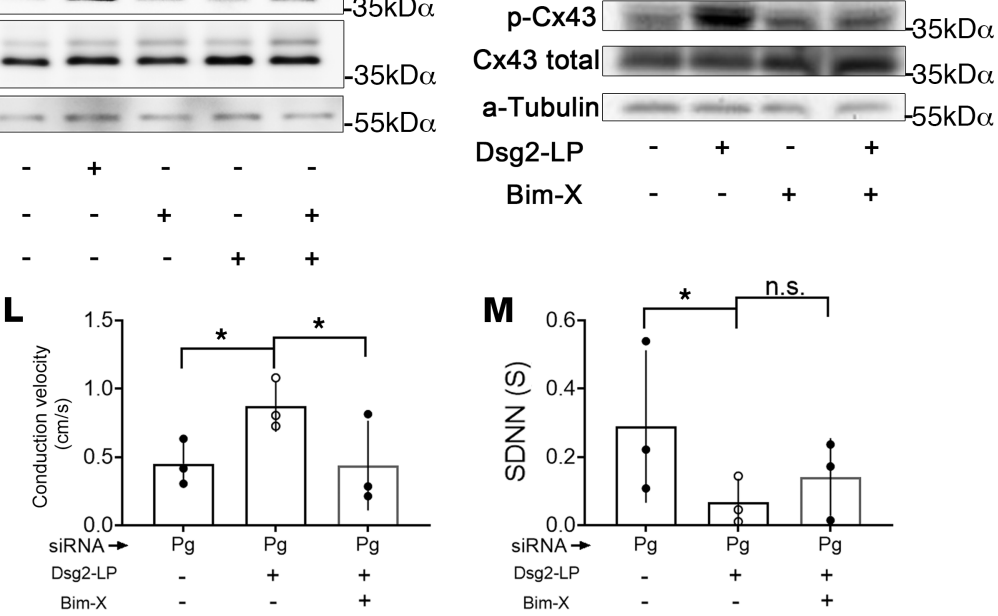

Figure 4. Stabilization of Dsg2 binding rescues irregular conduction of excitation and arrhythmia caused by impaired desmosomes. (A) Representative MEA heatmaps of transversal slices of WT and Pg-KO hearts treated with Dsg2-LP for 1 hour showing the delay of spontaneous excitations relative to a reference electrode $\left(^{*}\right)$. Colors correspond to the delay times of excitation given in the scale. Distance between neighboring electrodes: $200 \mu \mathrm{m}$. (B) Corresponding analysis of conduction velocity measured in cardiac slices as shown in $\mathbf{A}$ (WT control $n=9$; WT Dsg2-LP $n=6$ from 3 mice; Pg-KO control $n=$ 11; Pg-KO Dsg2-LP $n=12$ from 5 mice). Two-way ANOVA with Holm-Šidák post hoc test was performed. (C and F) MEA heatmaps from spontaneously contracting HL-1 cardiomyocytes treated with Dsg2-LP. Pg and Dsg2-levels were reduced by siRNA (Pg-siRNA, Dsg2-siRNA; n.t.-siRNA served as control). Distance between neighboring electrodes: $200 \mu \mathrm{m}$. (D, E, G, and $\mathbf{H}$ ) Corresponding analysis of conduction velocity and SDNN of MEAs as described in C (control $n=5 ; \operatorname{Trp} n=5 ; \operatorname{Trp}+\operatorname{Dsg} 2-\mathrm{LP} n=4 ;$ Dsg2-LP $n=5$ ), and (F) (n.t.-siRNA $n=5 ;$ Pg-siRNA $n=4 ;$ Pg-siRNA + Dsg2-LP $n=3 ;$ Dsg2-siRNA $n=5 ;$ Dsg2-siRNA + Dsg2-LP $n=3$ ). One-way ANOVA with Bonferroni's post hoc test was performed. (I and J) Western blot analysis shows phosphorylation of Cx43 at serine 368 ( $\mathrm{pC} \times 43$ Ser368) in response to PKC activation by PMA and PKC inhibition by Bim-X or Dsg2-LP in HL-1 (I) and WT mice ventricular cardiac slices (J). Values in top row display mean of densitometric analysis \pm SD. Total $C \times 43$ was used as a reference protein. $\alpha$-Tubulin ( $\alpha$-Tub) served as loading control ( $n=$ 4). (K-M) MEAs of Pg-reduced HL-1 cardiomyocytes treated with Bim-X and Dsg2-LP with corresponding analysis of conduction velocity and SDNN ( $n=3$ ). ${ }^{*} P<0.05$. One-way ANOVA with Bonferroni's post hoc test.

Dsg2-LP enhances Dsg2-mediated binding force on cardiomyocytes. As the next step, AFM measurements were performed on living cardiomyocytes to evaluate Dsg2-mediated binding and the effect of Dsg2-LP on a cellular level. For these experiments, a Dsg2-coated tip was probed at the area of cell-cell contact of 2 adjacent cardiomyocytes (Supplemental Figure 6A). The topography of the cell surface and the interaction properties, including binding event localization of the tip-bound molecule, can be determined simultaneously in 


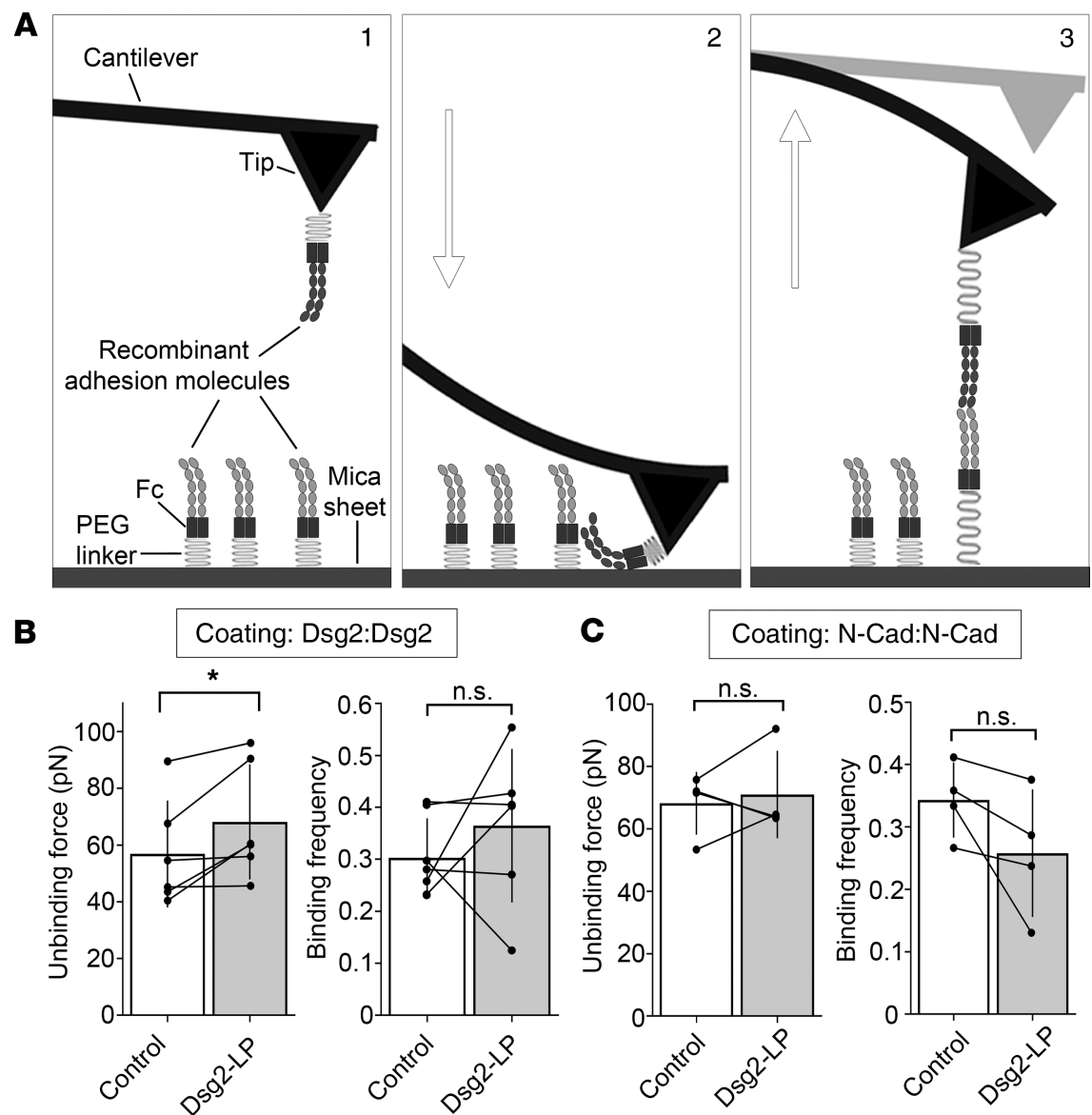

Figure 5. Dsg2-LP stabilizes Dsg2 binding in vitro. (A) Schematic of atomic force microscope (AFM) force mapping setup. The tip of a cantilever functionalized with the extracellular domains of adhesion molecules via a flexible PEG linker (step 1) is repeatedly extended to (step 2) and retracted from (step 3) a functionalized surface. Interaction of the tip- and surface-bound proteins is detectable by cantilever deflection. (B and $\mathbf{C}$ ) Analysis of unbinding force and binding frequency of cell-free AFM measurements with Dsg2- or N-Cad-coated tips probed on mica sheets with respective coatings. Every dot in the right panel represents the mean value of 1 independent experiment, bars indicate mean $\pm \mathrm{SD}$, and black lines connect paired values. $n=6$ for Dsg2; $n=5$ for N-Cad. n.s., $P \geq 0.05,{ }^{*} P<0.05$. Two-tailed paired Student's $t$ test.

this setup. In HL-1 cardiomyocytes, addition of Dsg2-LP induced an increase in the mean interaction force of Dsg2-mediated bindings from $27.3 \pm 0.26 \mathrm{pN}$ to $30.9 \pm 0.36 \mathrm{pN}$ while the binding frequency and localization of binding events at the measured area remained unchanged (Figure 6, A-C). Comparable results with elevated binding force from $39.1 \pm 0.39 \mathrm{pN}$ to $43.2 \pm 0.54 \mathrm{pN}$ were obtained at the cell-cell contacts of cardiomyocytes isolated from neonatal WT mouse hearts (Figure 6, D-F). AFM force mapping on cardiomyocytes derived from mice bearing a cardiac-specific Dsg2 deletion (Supplemental Figure 6B) revealed no changes in force and localization of Dsg2-mediated interactions compared with WT (Figure 6, G-I). This indicates interaction partners of Dsg2 in addition to homophilic binding as proposed before (17). However, the Dsg2-mediated binding force was significantly reduced to $28.8 \pm 0.48 \mathrm{pN}$ on Dsg2-KO compared with $36.6 \pm 0.72 \mathrm{pN}$ measured on WT cells. On Dsg2-KO cardiomyocytes, Dsg2-LP was not efficient to significantly increase Dsg2-mediated binding force, with $39.7 \pm 0.68 \mathrm{pN}$ compared with $38.4 \pm 0.65 \mathrm{pN}$ (Figure 6 , J-L). Together these data demonstrate that Dsg2-LP is sufficient to specifically stabilize Dsg2-mediated binding in vitro and on living cardiomyocytes.

Dsg2-LP enhances Dsg2 oligomer formation. Because we observed that Dsg2-LP treatment stabilized cell cohesion and enhanced Dsg2 binding on the molecular level, we investigated whether Dsg2-LP would affect Dsg2 clustering. Thus, we used sulfo-ethylene glycol bis(sulfosuccinimidyl succinate) (sulfo-EGS) cross-linking to test whether Dsg2-LP leads to oligomer formation in the cell membrane of HL-1 cells, as shown previously for other desmosomal cadherins (22). Western blot analyses 
A

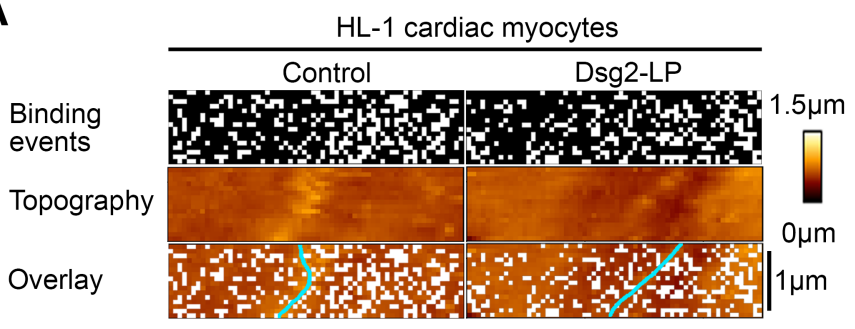

D

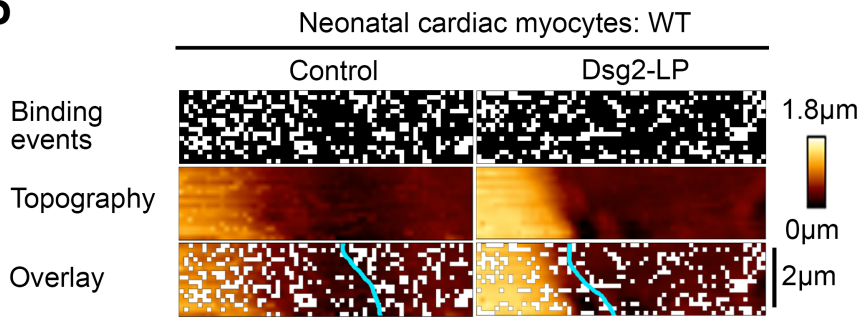

G

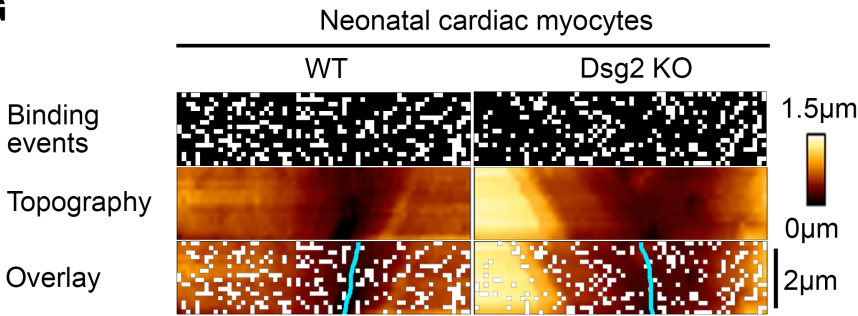

J

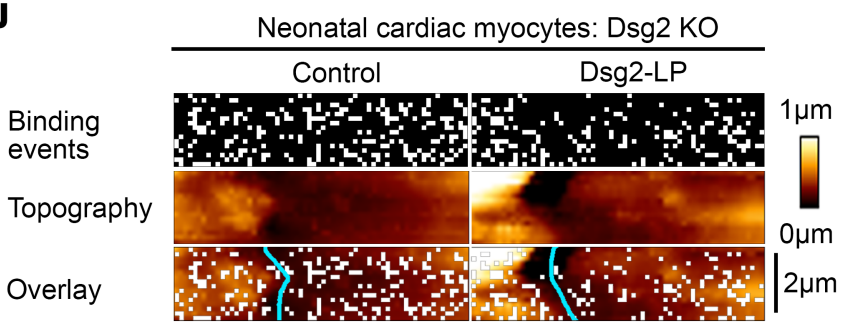

B

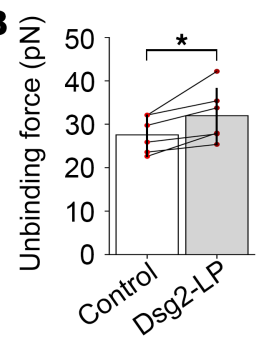

E
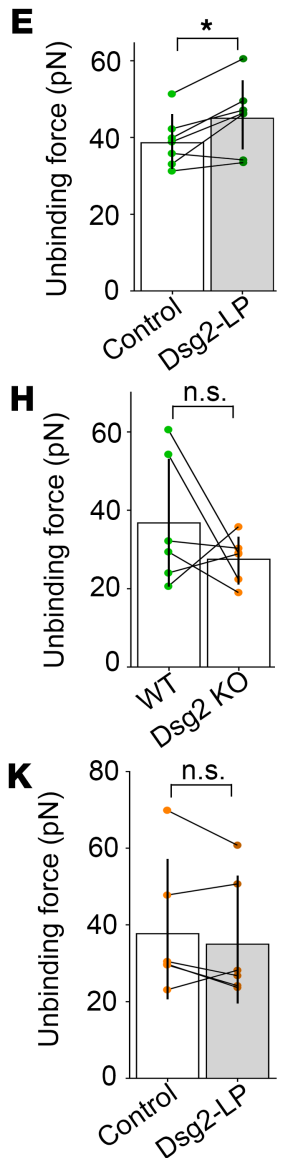

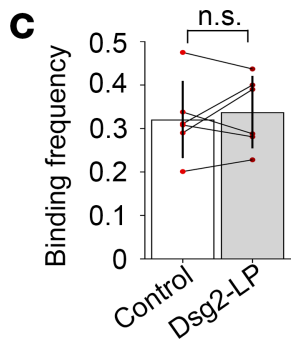

$\mathbf{F}$
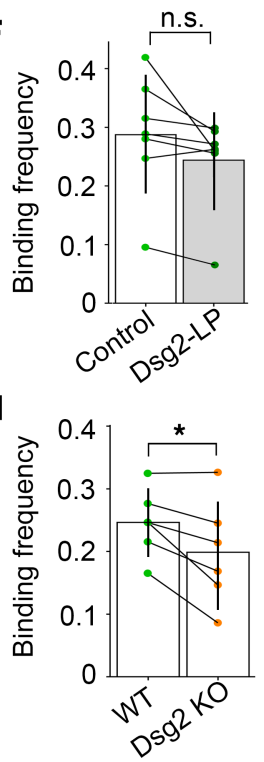

$\mathbf{L}$

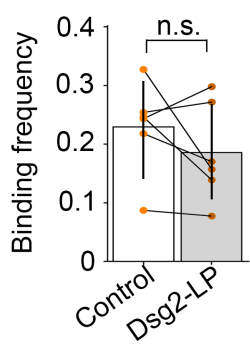

Figure 6. Dsg2-LP stabilizes Dsg2-mediated binding on living cardiomyocytes. Representative binding event and topography images of AFM force mapping performed with a Dsg2-coated tip on HL-1 cardiomyocytes (A) or cardiomyocytes isolated from Dsg2-KO mice or WT littermates (D, G, and J) treated with Dsg2-LP 30-90 minutes. Cyan line indicates cell-cell border. Every white pixel represents 1 binding event at the respective location. (A) $n=5$ independent experiments, scale bar: $1 \mu \mathrm{m}$. (D) $n=7$ mice, (C) $n=6$ mice per phenotype, and (J) $n=6$ mice; scale bar: $2 \mu \mathrm{m}$. Unbinding forces (B, E, H, and K) and binding frequency (C, F, I, and $\mathbf{L}$ ) of Dsg2-mediated binding events corresponding to respective panels on the left. Every dot represents the mean value of 1 independent experiment, bars indicate mean $\pm S D$, and black lines connect paired values. ${ }^{*} P<0.05$. Two-tailed paired Student's $t$ test was performed.

detected that Dsg2-LP treatment enhanced the formation of Dsg2 oligomers but not of N-Cad oligomers (Figure 7). This result confirmed that Dsg2-LP had specific effects on Dsg2 oligomer formation, which may contribute to enhanced cell cohesion.

\section{Discussion}

Based on the crystal structures of N-Cad (16) and Dsg2 (17), we designed the new linking peptide Dsg2-LP to cross-link Dsg2 molecules at extracellular domain 1. According to structure modeling, Dsg2-LP was designed to stabilize Dsg2 cis interaction, while trans interaction is assumed to be mediated by the Trp swap, describing the reciprocal insertion of Trp2 residues (4). Our data show that Dsg2-LP increased the binding forces of Dsg2-mediated interactions in vitro as well as on living cardiomyocytes and enhanced Dsg2 oligomerization. The peptide was sufficient to rescue cell-cell adhesion impaired by depletion of desmosomal molecules or excess of the amino acid Trp, known to disrupt the Trp swap (4). We believe that Dsg2-LP was protective 
A

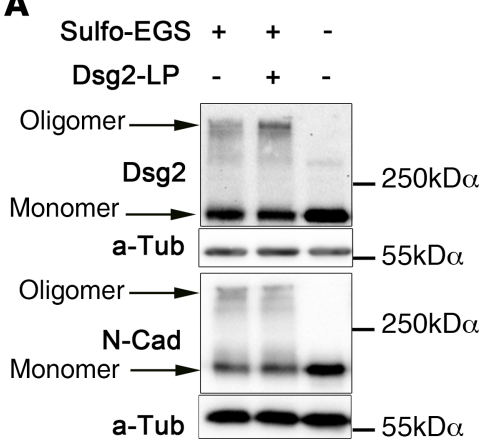

B

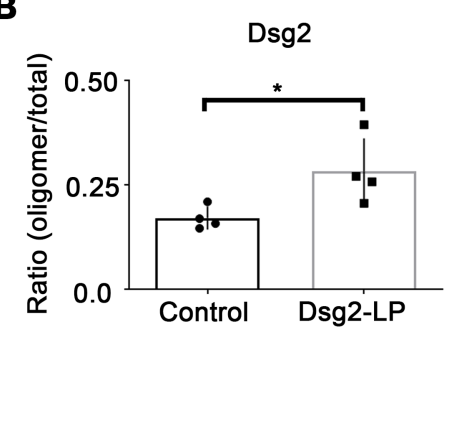

C

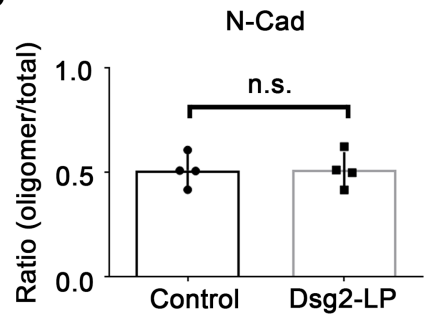

Figure 7. Dsg2-LP enhances formation of Dsg2 oligomers. (A) Membrane-impermeable cross-linking with sulfo-EGS performed after 24 -hour treatment with Dsg2-LP in HL-1 cells. Western blot analyses were done for Dsg2 and N-Cad. $\alpha$-Tubulin ( $\alpha$-Tub) served as loading control. Representative image of 4 repeated experiments. (B) Densitometric quantification of Dsg2 and N-Cad bands showing the ratio of cross-linked proteins to total protein. $n=4$. ${ }^{*} P<$ 0.05. Two-tailed paired Student's $t$ test with 95\% confidence levels was performed.

in HL-1 cells depleted of Pg and Dsg2 because both Pg- and Dsg2-siRNA did not completely abolish Dsg2 expression, which was also revealed by the Western blot (Figure 3, A and B). Thus, it is conceivable that adhesion of remaining Dsg2 molecules may be stabilized by Dsg-LP. This is in accordance with previous studies demonstrating that similar peptides interacting with Dsg3 or VE-cadherin rescue keratinocyte cohesion challenged with pemphigus autoantibodies or inflammatory endothelial barrier breakdown, respectively $(13,15)$. This effect of Dsg2-stabilizing peptides to increase cell-cell adhesion in the presence of adhesion-disturbing agents, such as autoantibodies, can be especially important because anti-Dsg2 autoantibodies were recently shown to be involved in the pathogenesis of AC (2). Furthermore, Dsg2-LP was efficient to rescue arrhythmia in an AC mouse model after 10-minute incubation. These data suggest that stabilization of Dsg2-mediated binding by a linking peptide has the capacity to acutely rescue ventricular arrhythmia right after short-term perfusion and may serve as a promising treatment option for patients with AC.

When we investigated the underlying mechanism, colocalization of Cx43 and Dsg2 was increased at cell-cell contacts by Dsg2-LP in AC mouse hearts or cultured cardiomyocytes treated with Trp. Furthermore, deficiency of Pg or Dsg2 or Trp treatment induced dysfunctional conduction of excitation with reduced conduction velocity paralleled to disrupted cell cohesion whereas Dsg2-LP was sufficient to acutely rescue these effects. These results indicate that impaired mechanical strength disturbs GJ functionality. This is consistent with the hypothesis of a molecular crosstalk of Cx43 with desmosomal components mediating GJ function (8-12, 23). Importantly, stabilizing Dsg2 binding rescued GJ function, highlighting the pivotal role of desmosomes for the structural and functional integrity of the ICD.

Phosphorylation of Cx43 at Ser368 by PKC was shown to regulate conduction velocity and selectivity of GJ permeability. This mechanism is suggested to protect the myocardium from arrhythmias under ischemic conditions $(6,7)$. We showed that Dsg2-LP induced Cx43 phosphorylation at Ser368 and that the protective effect of Dsg2-LP on excitation propagation after Pg depletion in vitro was dependent on PKC signaling. This suggests that phosphorylation of $\mathrm{Cx} 43$ at the PKC site Ser638 with subsequent alteration in GJ permeability and conduction velocity may contribute to the abrogation of arrhythmia and conduction irregularities induced by disturbed cohesion. However, in Pg-KO cardiac slices total Cx43 levels and membrane localization were reduced in accordance with already published data $(18,24)$, and Dsg-LP failed to significantly enhance $\mathrm{Cx} 43$ phosphorylation, indicating that $\mathrm{Cx} 43$ relocalization was not dependent on phosphorylation of $\mathrm{Cx} 43$ on Ser368. Taken together, PKC signaling may enhance cardiomyocyte excitation propagation indirectly via strengthening adhesion as shown before (8) or via other effects. Thus, a combination of enhanced Dsg2 adhesion and protective PKC-dependent signaling may account for the positive effect of Dsg2-LP on GJ function. However, interplay with ephaptic conduction of excitation via the cardiac sodium channel complex, which was shown to be modulated by adhesion-inhibiting peptides (25), cannot be ruled out.

In summary, we demonstrate that stabilization of Dsg2 binding by Dsg2-LP is efficient to acutely rescue arrhythmia in an AC mouse model and restore dysfunctional conduction of excitation in response to impaired desmosome integrity. Up to now, only symptomatic treatment options have been available to prevent ventricular arrhythmias and sudden cardiac death in patients with AC (1). Therefore, directly targeting 
impaired cardiomyocyte cohesion with consecutive improvement of GJ function would serve as a specific treatment alternative and a first step toward precision medicine.

\section{Methods}

Modeling of the Dsg2 extracellular domain. At the time the peptide was designed, structure data of Dsg2 were limited to a nuclear magnetic resonance (NMR) solution structure analysis of the first extracellular domain (EC) of Dsg2 (Protein Data Bank [PDB] entry 2YQG). Therefore, a theoretical cis- and trans-interacting Dsg2 assembly was built from 2 crystal structures available for N-Cad. The PDB entry 1NCH (16) represents a potential assembly of 2 cis-interacting EC1s, while the PDB entry 1 NCI represents a potential assembly of 2 trans-interacting EC1s. The amino acid sequences of the EC1 of human Dsg2 were aligned to those of $\mathrm{N}$-Cad moieties of both assemblies, and an assembly for cis and trans interaction was built by replacing the amino acids of N-Cad in both templates, $1 \mathrm{NCH}$ and $1 \mathrm{NCI}$, with those of human Dsg2 using the ProteinDesign tool of the software package Quanta2008 (MSI Accelrys). Deletions and insertions in Dsg2 resulting from difference from the N-Cad template were modeled manually using the NMR structures (20 structures are included in the ensemble) of Dsg2 to provide guidance for the modeling of the most likely backbone conformations. The van der Waals overlap between side chain atoms was removed by side chain rotamer searches using the XBuild tool of the software Quanta2008. The model comprising 3 Dsg2 EC1s arranged in 1 cis and 1 trans interaction was subsequently refined by a stepwise energy minimization (200 steps each) using only geometrical energy terms (no electrostatic) with first restraining all backbone atoms with a strong harmonic potential (force constant $50[\mathrm{kcal} / \mathrm{mol}] / \AA$ ) and then lowering the restraints on the backbone atoms to 10,5 , and $0(\mathrm{kcal} / \mathrm{mol}) / \AA$ to maintain the secondary structure of the template.

Design of Dsg2-IP and Dsg2-LP. Dsg2-IP was modeled by analyzing the interfaces of the cis- and trans-interacting assemblies. While the trans interaction predicted a peptide segment harboring the known tryptophan motif at the N-terminus of EC1 (Ala1 to Pro6), from the cis interaction interface, 2 peptide segments could be identified, either Asn56 to Gly60, which is the $\beta 4 \beta 5$ loop, or the $\beta 6 \beta 7$ loop comprising Ala82 to Val90. Because the latter showed more intimate hydrophobic as well as polar contacts, this $\beta$-turn forming loop was used as the basis for Dsg2-IP. The 2 residues Ala82 and Va190 were replaced by cysteines, thereby allowing the linear Dsg2-IP to be converted into a cyclic peptide exhibiting a higher stability and forming a conformationally constrained peptide loop, as observed in the 3D model. In addition, we exchanged Leu83 and Asn89 to phenylalanine because our model suggested this would result in enhanced hydrophobic interactions, thereby strengthening the binding of the Dsg2-IP to Dsg2. The final monomeric Dsg2-IP had thus the sequence Ac-CFDARGNFC-NH . $_{2}$ Dsg2-LP was derived by altering the sequence to Ac-CFDARGNFC-aminohexan-CFDARGNFC-NH $\mathrm{N}_{2}$ with disulfide bonds between the first and the second and the third and the forth cysteine residue. This then yielded the dimeric Dsg2-LP enabling noncovalent cross-linking of Dsg2 moieties.

Cell culture. HL-1 cells, a murine atrial cardiomyocyte cell line, were provided by William C. Claycomb (Department of Biochemistry and Molecular Biology, Louisiana State University Health Sciences Center, New Orleans, Louisiana, USA) and cultured according to his instructions (26). Cell culture reagents were purchased from MilliporeSigma. Cells were maintained in Claycomb Medium (51800C) supplemented with 10\% fetal bovine serum (F2442), $100 \mu \mathrm{M}$ norepinephrine, penicillin/streptomycin, and $2 \mathrm{mM}$ L-glutamine at $37^{\circ} \mathrm{C}, 5 \% \mathrm{CO}_{2}$, and $95 \%$ humidity. For immunostaining, dissociation assay, and Western blot analysis, cells were seeded at 25,000 cells $/ \mathrm{cm}^{2}$ and incubated for 7 days on glass coverslips or plates coated with $0.02 \%$ gelatin and $25 \mu \mathrm{g} / \mathrm{mL}$ fibronectin. After seeding for experiments, cells were incubated in Claycomb Medium without norepinephrine to avoid basal adrenergic stimulation and an additional $1.8 \mathrm{mM} \mathrm{CaCl}_{2}$ to ensure $\mathrm{Ca}^{2+}$-dependent cadherin binding.

Mouse models and preparation of cardiomyocytes and cardiac slices. Animal handling and sacrifice were in accordance with guidelines of the European Commission and local university regulations of LMU Munich and RWTH Aachen University. For generation of heart-specific Pg (18) or Dsg2 (10) deficiency in mice, the loxP/Cre system with Cre under the $\alpha$-myosin heavy chain promoter was used as described before. Both mouse strains develop an AC-like phenotype with progressive cardiac hypertrophy, ventricular dilatation, and fibrosis of the heart muscle $(10,18,24,27)$. Genotype of all animals was evaluated by PCR. Murine cardiac slices were prepared as previously described (18) and mounted on an MEA setup as described below. Alternating slices were used for control and Dsg2-LP treatment. Age- and sex-matched mice were used. Neonatal cardiomyocytes were isolated and maintained according to a published protocol (28) with seeding on laminin-coated (MilliporeSigma, L2020) glass coverslips and 
incubation at $37^{\circ} \mathrm{C}, 5 \% \mathrm{CO}_{2}$, and $95 \%$ humidity for 3 to 6 days till proliferation of cardiomyocytes and establishment of intercellular contacts.

siRNA-mediated knockdown. ON-TARGET plus SMARTpool mouse Dsg2-, Jup-, or nontargeting siRNA (Dsg2-, Pg-, and n.t.-siRNA) (Dharmacon, Thermo Fisher Scientific; L-042514-01-005, L-040316-01-005, and D-001810-10-05, respectively) was transferred into HL-1 cells by electroporation (4D-Nucleofector, Lonza, AAF-1002Y) according to the manufacturer's instructions. Confluent HL-1 cells were trypsinized, counted, and electroporated in Amaxa SF solution (Lonza) with $150 \mu \mathrm{g} / \mathrm{mL}$ siRNA at a concentration of $4 \times 10^{7}$ cells $/ \mathrm{mL}$ with pulse EN150. Experiments were performed 72 hours after electroporation. Transfection with n.t.-siRNA was performed in parallel. For all experiments, knockdown efficiency was confirmed by Western blot analysis.

Antibodies and mediators. The following primary antibodies were used for Western blot (WB) analysis or immunostaining evaluated by confocal or STED microscopy: monoclonal mouse anti-Dsg1/2 for WB and confocal microscopy (Progen Biotechnik, 61002), polyclonal rabbit anti-Dsg2 for STED (Progen Biotechnik, 610121), monoclonal mouse anti-Pg for WB and confocal microscopy (Progen Biotechnik, 61005), monoclonal mouse anti- $\alpha$-tubulin for WB (Abcam, ab7291), polyclonal rabbit anti-Cx43 for confocal microscopy and WB (MilliporeSigma, SAB4501175), monoclonal mouse anti-Cx43 for STED (Merck KGaA, MAB3067), phospho-Cx43 Ser368 for WB (Santa Cruz Biotechnology Inc., sc-101660-r), monoclonal mouse anti-N-cadherin (BD Biosciences, 610921), and monoclonal mouse anti-Dp (Progen Biotechnik, 61003). Secondary antibodies were goat anti-rabbit IgG or goat anti-mouse IgG and IgM antibodies conjugated to peroxidase (11-035-003 and 115-035-068) for WB or conjugated to Cy2 (111-225-144 and 115-225-166) or Cy3 (111-165-003 and 115-165-068) for confocal microscopy (all Dianova) or STAR red goat anti-rabbit IgG (Abberior GmbH, 2-0012-011-9) or Alexa Fluor 594 goat anti-mouse IgG (Abcam, ab150116) for STED. For blocking of Dsg2-mediated interaction in AFM experiments, monoclonal mouse anti-Dsg2 (10G11, Progen Biotechnik, 61059) was applied at $1 \mu \mathrm{g} / \mathrm{mL}$ and incubated for 30 minutes. Trp (MilliporeSigma) was dissolved in PBS at $10 \mathrm{mM}$, pH was adjusted to 7.4 , and aliquots were stored at $-20^{\circ} \mathrm{C}$ and used in working concentrations of $400 \mu \mathrm{M}$ for 24 hours. Production of Dsg2-LP and VE-Cad-LP with corresponding Dsg2-IP was commissioned to Bachem. All peptides were dissolved at 10-mM concentration in DMSO, stored at $-20^{\circ} \mathrm{C}$, and applied at concentrations of $20 \mu \mathrm{M}$ for 24 hours or as indicated. Carbenoxolone (Abcam, ab143590) was dissolved in $\mathrm{H}_{2} \mathrm{O}$ and used in concentrations of $20 \mu \mathrm{M}$ for 24 hours to inhibit GJs. Bim-X (Enzo Life Sciences, 270-057-M001) was dissolved in DMSO and used in concentrations of 1 $\mu \mathrm{M}$ for 24 hours (HL-1 cells) and 1 hour (mouse ventricular cardiac slices) to inhibit PKC activity. PMA (MilliporeSigma, P6139) was dissolved in DMSO and used in concentrations of $100 \mathrm{nM}$ for 24 hours to activate PKC. EGTA (VWR International, 0732) was dissolved in $\mathrm{H}_{2} \mathrm{O}$ and used in concentrations of $5 \mathrm{mM}$ for chelation of $\mathrm{Ca}^{2+}$. All solutions applied in cell culture were sterile filtrated before use.

Immunostaining. Immunostaining of cultured cardiomyocytes and heart tissue was performed as previously described (18). After incubation with corresponding reagents, cells were washed with PBS, fixed in 2\% paraformaldehyde in PBS for 10 minutes, and permeabilized with $0.1 \%$ Triton X-100 in PBS for 5 minutes, following blocking of unspecific binding sites with $3 \%$ bovine serum albumin/10\% normal goat serum in PBS for 30 minutes. Primary antibodies diluted in PBS were incubated at $4^{\circ} \mathrm{C}$ in a humid chamber overnight. Cardiac tissue was snap-frozen in liquid nitrogen and cut with a cryostat (Cryostar NX70, Thermo Fisher Scientific) in 7- $\mu \mathrm{m}$-thick sections. Probes were then heated to $37^{\circ} \mathrm{C}$ for 8 minutes, washed in PBS, and fixed in 2\% PFA for 10 minutes. Then slices were permeabilized with 1\% Triton X-100 for 60 minutes, washed in PBS, and blocked with 3\% bovine serum albumin/10\% normal goat serum in PBS for 60 minutes. Species-matched secondary antibodies were applied in PBS at room temperature in a humid chamber for 1 hour. Glass coverslips were mounted using $1.5 \%$ of the antifading reagent $N$-propyl gallate (MilliporeSigma, P3640000) and 60\% glycerol in PBS for confocal microscopy or ProLong Diamond Antifade Mountant (Invitrogen, Thermo Fisher Scientific, P36965) for STED microscopy. Staining was evaluated using a Leica SP5 confocal microscope equipped with a $\times 63$ oil objective using LAS-AF software for image acquisition. STED images were obtained using an expert line setup from Abberior (Abberior Instruments) equipped with a $\times 100$ oil objective using Imspector image acquisition software (Abberior Instruments). ImageJ software (NIH) was applied for image analysis.

Colocalization of Dsg2 and Cx43 staining was analyzed using ImageJ software. Single images obtained by STED microscopy were transferred to 8-bit mode and merged into stacks while keeping the source image. For quantitative analysis, 4 ICDs per mouse per condition were analyzed. For 2 corresponding images of both Dsg2 and Cx43 staining, respectively, the intercalated disc was marked as the region of 
interest by circling the stained area manually. After that colocalization threshold, analysis was applied while not using constant intensity for colocalized pixels, and the number of colocalized voxels/pixels was obtained to compare the amount of colocalization of Dsg2 and Cx43 staining in different conditions. The colocalized points appear in white as shown in Figure 2A.

Sulfo-EGS cross-linking. Oligomerization of Dsg2 and N-Cad at the cell membrane was investigated by using the membrane-impermeable cross-linker sulfo-EGS (21566, Pierce Biotechnology, Thermo Fisher Scientific). Sulfo-EGS was added to cells 24 hours after Dsg2-LP treatment at a concentration of $2 \mathrm{mM}$ for 30 minutes at room temperature. TBS $(50 \mathrm{mM})$ was added to stop the reaction, and WBs were performed to detect cross-linked products.

WB analysis. WB analysis was performed using standard procedures. HL-1 cells treated with indicated reagents were washed with PBS and scratched in ice-cold SDS-lysis buffer (25 mM HEPES, $2 \mathrm{mM}$ EDTA, $25 \mathrm{mM} \mathrm{NaF}, 1 \%$ SDS, pH 7.4) supplemented with protease inhibitor cocktail (cOmplete, Roche, 11697498001). Lysates were sonicated and protein content was estimated using the BCA method (Thermo Fisher Scientific, 23225). Protein samples were denatured in Laemmli buffer at $95^{\circ} \mathrm{C}$ for 5 minutes before separation on $10 \%$ SDS-polyacrylamide gels. Proteins were transferred on nitrocellulose membranes (Novex, Thermo Fisher Scientific) using the wet-blot method and blocked in 5\% low-fat milk in TBS-T (20 mM Tris base, $137 \mathrm{mM} \mathrm{NaCl}, 0.0475 \%$ Tween, $\mathrm{pH}$ 7.6) buffer. Primary antibody incubation was performed in 5\% low-fat milk or bovine serum albumin in TBS-T according to the manufacturer's instructions at $4^{\circ} \mathrm{C}$ overnight. Species-matched peroxidase-conjugated secondary antibodies were incubated at room temperature for 2 hours in 5\% low-fat milk in TBS-T and developed using the ECL method (0.5\% luminol, $0.25 \%$ para-coumaric acid, $0.03 \%$ hydrogen peroxide, $0.1 \mathrm{M}$ Tris- $\mathrm{HCl}$ ) for chemiluminescence protein band visualization. Band densitometric analysis was performed using ImageJ software.

Dissociation assay. HL-1 cells were treated as indicated and grown to confluence. Cell monolayers were washed with PBS and incubated with $200 \mu \mathrm{L}$ dissociation buffer (liberase DH $0.065 \mathrm{U} / \mathrm{mL}$, MilliporeSigma, 5401054001; dispase II $2.5 \mathrm{U} / \mathrm{mL}$, MilliporeSigma, D4693 in HBSS) per well at $37^{\circ} \mathrm{C}$ till detachment of the HL-1 cell monolayer from the well bottom. After detachment, the dissociation buffer was replaced with $350 \mu \mathrm{L}$ HBSS, and the monolayers were mechanically stressed by shaking on an orbital shaker. The total number of resulting fragments per well was determined using a binocular stereo microscope (Leica). Fragments were counted if they were clearly visible at 1.25 -fold magnification. The number of fragments is an indirect measure for intercellular cohesion.

Langendorff heart preparation. Adult murine hearts were used for Langendorff heart preparations on a commercially available apparatus (AD Instruments) and analyzed using LabChart7 software as described previously (19). Animal handling and sacrifice were in accordance with guidelines of the European Commission and local university regulations. Pg-KO mice and WT littermates, 12 weeks old, were obtained from the institutes' animal facilities, were sacrificed by cervical dislocation, and underwent subsequent preparation of the hearts in less than 5 minutes. Hearts were perfused in the retrograde Langendorff mode with heparin-free carbogen-gassed Krebs-Henseleit buffer supplemented with $18.8 \mathrm{nM}$ norepinephrine at $37^{\circ} \mathrm{C}$ with $60 \mathrm{mmH}_{2} \mathrm{O}$ constant pressure. ECG was measured via needle electrodes, and pulse pressure was determined via the aortic cannula. After recording of baseline for 10 minutes, Dsg2-LP or VE-Cad-LP at a concentration of $20 \mu \mathrm{m}$ dissolved in DMSO and Krebs-Henseleit buffer were added to the perfusion solution for the indicated time using a perfusion pump.

Atomic force microscopy. For measuring simultaneously topography, distribution of transmembrane adhesion molecules, and their binding properties, a Nanowizard III atomic force microscope (JPK Instruments) mounted on an optical fluorescence microscope (Axio Observer D1, Carl Zeiss) was used. Recombinant Dsg2 was purified from cell culture supernatant of stably Dsg2-Fc-expressing Chinese hamster ovary cells as described previously (29). N-Cad-Fc was purchased from R\&D Systems (Bio-Techne). Flexible Si3N4 AFM cantilevers (MLCT probes, Bruker) were coated with bifunctional PEG spacers (AcetalPEG-NHS, Gruber Lab, Institute of Biophysics, Linz, Austria) to link recombinant molecules (at concentrations of $0.1 \mathrm{mg} / \mathrm{mL}$ ) as described before (30). For experiments, the pyramid-shaped D-tip (nominal spring constant: $0.03 \mathrm{~N} / \mathrm{m}$ for HL-1 cells) or C-tip (nominal spring constant: $0.01 \mathrm{~N} / \mathrm{m}$ for murine neonatal cells) was used. To create AFM images and perform adhesion measurements, SPM Control v.4 software (JPK Instruments) was used. For visualization of cells, a bright-field image was acquired with a $\times 63$ objective and an overview AFM topography image was created using a force curve-based quantitative imaging mode with the following settings: set point $0.2 \mathrm{nN}$, pulling speed $50 \mu \mathrm{m} / \mathrm{s}$, and z-length $1500 \mathrm{~nm}$. 
For adhesion measurements, a cell-cell border-containing region was selected, and measurements were performed on a $64 \times 16$ pixel grid in force-mapping mode with the following settings: set point $0.2 \mathrm{nN}$, extend speed $5 \mu \mathrm{m} / \mathrm{s}$ (for HL-1) or $15 \mu \mathrm{m} / \mathrm{s}$ (for murine neonatal cells), and extend delay 0.1 second. Each pixel acquired while scanning the selected region of interest contains the information of 1 force-distance curve. For each of these pixels, the tip repeatedly approaches the cell surface, indents the cell membrane until the defined set point is reached, and retracts again. During this movement, the deflection of the flexible cantilever is detected by a laser beam with regard to its relative position over the cell surface, creating a force-distance plot. In the case of a binding event, the tip is held back by the interacting molecules, and the cantilever is deflected downward. When the force exerted by the pulling tip exceeds the binding force, the bond ruptures, and the cantilever rapidly bends back into the neutral position (unbinding event) and is further retracted to a predefined height before a novel approach is started 1 pixel lateral to the previous one. The amount of the cantilever deflection can be converted into forces acting on the cantilever using Hooke's law. The spring constant $\mathrm{k}$ was determined separately by the thermal noise method for each tip (31). The amplitude of the "jump" in the force plot during an unbinding event represents the strength of the bond. For all experiments, the same cell-cell border was measured before and after addition of Dsg2LP $20 \mu \mathrm{M}$. During measurements, contraction of murine neonatal cells was inhibited with 2,3-butanedione $20 \mathrm{mM}$ (MilliporeSigma, B85307). Measurements for cell-free conditions were performed in HBSS containing $1.2 \mathrm{mM} \mathrm{Ca}^{2+}$ at $37^{\circ} \mathrm{C}$ on cleaved mica sheets (SPI Supplies) functionalized with $\mathrm{Fc}_{\mathrm{c}}$ constructs as described above for AFM probes.

MEA analysis. For MEA, we used the MEA2100-60-System equipped with 60MEA200/30iR-Ti-gr electrode chambers (both Multichannel Systems). For seeding of HL-1 cells, electrode chambers were precoated with fetal bovine serum and coated with $0.02 \%$ gelatin per $25 \mu \mathrm{g} / \mathrm{mL}$ fibronectin. HL- 1 cells were seeded at 550,000 cells/chamber, or at 750,000 cells/chamber if nucleofection was performed, and grown to confluence over 3 days. Application of the indicated mediators was performed for 24 hours, and medium supplemented with these mediators was changed immediately before starting the measurements. Then, $300-\mu \mathrm{m}$-thick cardiac slices were transferred to cardiac slice medium consisting of DMEM F12 (1:1) medium supplemented with $25 \%$ fetal bovine serum, $1 \%$ minimum essential medium nonessential amino acids, $3 \%$ sodium pyruvate, $1 \%$ insulin/transferrin/selenium (all purchased from Life Technologies), $2 \mathrm{mM} \mathrm{L-glu-}$ tamine (MilliporeSigma, G3126), and $10 \mathrm{U} / \mathrm{L}: 10 \mu \mathrm{g} / \mathrm{mL}$ penicillin and streptomycin at $37^{\circ} \mathrm{C}, 5 \% \mathrm{CO}_{2}$, for 30 minutes. During this time, slices were treated with vehicle (DMSO) or $20 \mu \mathrm{M}$ Dsg2-LP for 1 hour. After treatment, cardiac slices were placed on MEA electrodes containing $250 \mu \mathrm{L}$ of cardiac slice medium, and measurements were performed with MC_Rack software 4.6.2 (Multichannel Systems). An electrode in the middle of the array was selected as the reference electrode, and electrical potential was recorded over a time of 90 seconds for HL-1 cells or 60 seconds for cardiac slices. Conduction of electrical signals between the cells was evaluated by calculating the mean time for propagation between 2 electrodes $(200 \mu \mathrm{m})$. Analysis was performed using heatmaps and tracings.

Hanging drop bead aggregation assay. The assay was carried out as described previously (32). In brief, protein G-coated beads (Dynabeads, Thermo Fisher Scientific, 10003D) were coated with recombinant Dsg2-Fc via covalent binding using the cross-linker dimethyl adipimidate (Thermo Fisher Scientific, 20660). Functionalized beads were aggregated in a hanging drop of HBSS on the lid of a culture dish at $37^{\circ} \mathrm{C}$ for 1 hour, followed by 1 hour of incubation with indicated mediators. After shaking on an orbital shaker at $1000 \mathrm{rpm}$ for 3 minutes, images of all drops were acquired, and a ratio was calculated from the area covered with beads and the area of total bead extent using ImageJ software.

Image analysis and processing. For image processing, Photoshop CS5 (Adobe) was used. Excel (Microsoft) and GraphPad Prism 7.04 (GraphPad Software Inc.) were applied for analyzing and creating graphs and plots. Immunostained images and WB bands were analyzed using ImageJ software. See complete unedited blots in the supplemental material. For analyzing AFM force-distance curves and processing AFM images, JPK data-processing software (JPK Instruments) was applied. All other applied acquisition and analysis software is mentioned in the respective Methods section.

Statistics. Results are expressed as mean values \pm SD. Statistical comparisons were performed using either 2-tailed Student's $t$ test for the comparison of 2 groups of samples or 1-way ANOVA or 2-way ANOVA with post hoc test in the case of multiple-group comparisons using GraphPad Prism 7.04 or 8. Significance was assumed for $P<0.05$. 


\section{Author contributions}

CS, BME, SY, AS, VR, EK, and TDM acquired data and analyzed the data; CS, BME, SY, AS, TDM, and JW drafted the manuscript and made critical revisions of the manuscript for important intellectual content; and SK and RL provided reagents. JW supervised and designed the research.

\section{Acknowledgments}

We thank Angelika Antonius, Kilian Skowranek, and Cathleen Plietz for technical assistance. This work was supported by the Deutsche Forschungsgemeinschaft (WA2474/11-1 to JW and via DFG-SPP1782 to JW and RL).

Address correspondence to: Jens Waschke, Institute of Anatomy and Cell Biology, Department I, Ludwig-Maximilians-Universität, Pettenkoferstraße 11, 80336 Munich, Germany. Phone: 49.89.2180.72602; Email: jens.waschke@med.uni-muenchen.de.

1. Corrado D, Link MS, Calkins H. Arrhythmogenic right ventricular cardiomyopathy. N Engl J Med. 2017;376(1):61-72.

2. Chatterjee D, et al. An autoantibody identifies arrhythmogenic right ventricular cardiomyopathy and participates in its pathogenesis. Eur Heart J. 2018;39(44):3932-3944.

3. Franke WW, Borrmann CM, Grund C, Pieperhoff S. The area composita of adhering junctions connecting heart muscle cells of vertebrates. I. Molecular definition in intercalated disks of cardiomyocytes by immunoelectron microscopy of desmosomal proteins. Eur J Cell Biol. 2006;85(2):69-82.

4. Al-Amoudi A, Frangakis AS. Structural studies on desmosomes. Biochem Soc Trans. 2008;36(pt 2):181-187.

5. Saffitz JE, Laing JG, Yamada KA. Connexin expression and turnover: implications for cardiac excitability. Circ Res. 2000;86(7):723-728.

6. Naitoh $\mathrm{K}$, et al. Roles of $\mathrm{Cx} 43$-associated protein kinases in suppression of gap junction-mediated chemical coupling by ischemic preconditioning. Am J Physiol Heart Circ Physiol. 2009;296(2):H396-H403.

7. Lampe PD, TenBroek EM, Burt JM, Kurata WE, Johnson RG, Lau AF. Phosphorylation of connexin43 on serine368 by protein kinase C regulates gap junctional communication. J Cell Biol. 2000;149(7):1503-1512.

8. Schinner C, Erber BM, Yeruva S, Waschke J. Regulation of cardiac myocyte cohesion and gap junctions via desmosomal adhesion. Acta Physiol (Oxf). 2019;226(2):e13242.

9. Oxford EM, Musa H, Maass K, Coombs W, Taffet SM, Delmar M. Connexin43 remodeling caused by inhibition of plakophilin-2 expression in cardiac cells. Circ Res. 2007;101(7):703-711.

10. Kant S, Holthöfer B, Magin TM, Krusche CA, Leube RE. Desmoglein 2-dependent arrhythmogenic cardiomyopathy is caused by a loss of adhesive function. Circ Cardiovasc Genet. 2015;8(4):553-563.

11. Patel DM, Dubash AD, Kreitzer G, Green KJ. Disease mutations in desmoplakin inhibit Cx43 membrane targeting mediated by desmoplakin-EB1 interactions. J Cell Biol. 2014;206(6):779-797.

12. Kam CY, et al. Desmoplakin maintains gap junctions by inhibiting Ras/MAPK and lysosomal degradation of connexin-43. J Cell Biol. 2018;217(9):3219-3235.

13. Spindler V, et al. Peptide-mediated desmoglein 3 crosslinking prevents pemphigus vulgaris autoantibody-induced skin blistering. J Clin Invest. 2013;123(2):800-811.

14. Stanley JR, Amagai M. Pemphigus, bullous impetigo, and the staphylococcal scalded-skin syndrome. $N$ Engl J Med. 2006;355(17):1800-1810.

15. Heupel WM, et al. Endothelial barrier stabilization by a cyclic tandem peptide targeting VE-cadherin transinteraction in vitro and in vivo. J Cell Sci. 2009;122(pt 10):1616-1625.

16. Shapiro L, et al. Structural basis of cell-cell adhesion by cadherins. Nature. 1995;374(6520):327-337.

17. Harrison OJ, et al. Structural basis of adhesive binding by desmocollins and desmogleins. Proc Natl Acad Sci U S A. 2016;113(26):7160-7165.

18. Schinner C, et al. Adrenergic signaling strengthens cardiac myocyte cohesion. Circ Res. 2017;120(8):1305-1317.

19. Schlipp A, et al. Desmoglein-2 interaction is crucial for cardiomyocyte cohesion and function. Cardiovasc Res. 2014;104(2):245-257.

20. Hartlieb E, Kempf B, Partilla M, Vigh B, Spindler V, Waschke J. Desmoglein 2 is less important than desmoglein 3 for keratinocyte cohesion. PLoS ONE. 2013;8(1):e53739.

21. Hinterdorfer P, Dufrêne YF. Detection and localization of single molecular recognition events using atomic force microscopy. Nat Methods. 2006;3(5):347-355.

22. Fuchs M, et al. Plakophilin 1 but not plakophilin 3 regulates desmoglein clustering. Cell Mol Life Sci. 2019;76(17):3465-3476.

23. Delmar M, McKenna WJ. The cardiac desmosome and arrhythmogenic cardiomyopathies: from gene to disease. Circ Res. 2010;107(6):700-714.

24. Li J, Swope D, Raess N, Cheng L, Muller EJ, Radice GL. Cardiac tissue-restricted deletion of plakoglobin results in progressive cardiomyopathy and activation of $\beta$-catenin signaling. Mol Cell Biol. 2011;31(6):1134-1144.

25 . Veeraraghavan $\mathrm{R}$, et al. The adhesion function of the sodium channel beta subunit $(\beta 1)$ contributes to cardiac action potential propagation. Elife. 2018;7:e37610.

26. Claycomb WC, et al. HL-1 cells: a cardiac muscle cell line that contracts and retains phenotypic characteristics of the adult cardiomyocyte. Proc Natl Acad Sci U S A. 1998;95(6):2979-2984.

27. Li D, et al. Restrictive loss of plakoglobin in cardiomyocytes leads to arrhythmogenic cardiomyopathy. Hum Mol Genet. 2011;20(23):4582-4596 
28. Ehler E, Moore-Morris T, Lange S. Isolation and culture of neonatal mouse cardiomyocytes. J Vis Exp. 2013;(79):50154.

29. Schlegel N, Meir M, Heupel WM, Holthöfer B, Leube RE, Waschke J. Desmoglein 2-mediated adhesion is required for intestinal epithelial barrier integrity. Am J Physiol Gastrointest Liver Physiol. 2010;298(5):G774-G783.

30. Ebner A, et al. A new, simple method for linking of antibodies to atomic force microscopy tips. Bioconjug Chem. 2007;18(4):1176-1184.

31. Slattery AD, Quinton JS, Gibson CT. Atomic force microscope cantilever calibration using a focused ion beam. Nanotechnology. 2012;23(28):285704

32. Vielmuth F, Hartlieb E, Kugelmann D, Waschke J, Spindler V. Atomic force microscopy identifies regions of distinct desmoglein 3 adhesive properties on living keratinocytes. Nanomedicine. 2015;11(3):511-520. 